\title{
Transverse Space-Charge Field-Induced Plasma Dynamics for Ultraintense Electron-Beam Characterization
}

\author{
R. Tarkeshian, ${ }^{1,2,{ }^{*}}$ J. L. Vay, ${ }^{3}$ R. Lehe,${ }^{3}$ C. B. Schroeder,${ }^{3}$ E. H. Esarey, ${ }^{3}$ T. Feurer, ${ }^{1}$ and W. P. Leemans ${ }^{3}$ \\ ${ }^{1}$ Universität Bern, Bern-3012, Switzerland \\ ${ }^{2}$ Paul Scherrer Institute, Villigen-5232, Switzerland \\ ${ }^{3}$ Lawrence Berkeley National Laboratory, Berkeley, California 94720, USA
}

(Received 15 December 2017; revised manuscript received 22 March 2018; published 10 May 2018)

\begin{abstract}
Similarly to laser or x-ray beams, the interaction of sufficiently intense particle beams with neutral gases will result in the creation of plasma. In contrast to photon-based ionization, the strong unipolar field of a particle beam can generate a plasma where the electron population receives a large initial momentum kick and escapes, leaving behind unshielded ions. Measuring the properties of the ensuing Coulomb exploding ions-such as their kinetic energy distribution, yield, and spatial distribution-can provide information about the peak electric fields that are achieved in the electron beams. Particle-in-cell simulations and analytical models are presented for high-brightness electron beams of a few femtoseconds or even hundreds of attoseconds, and transverse beam sizes on the micron scale, as generated by today's free electron lasers. Different density regimes for the utilization as a potential diagnostics are explored, and the fundamental differences in plasma dynamical behavior for e-beam or photon-based ionization are highlighted. By measuring the dynamics of field-induced ions for different gas and beam densities, a lower bound on the beam charge density can be obtained in a single shot and in a noninvasive way. The exponential dependency of the ionization yield on the beam properties can provide unprecedented spatial and temporal resolution, at the submicrometer and subfemtosecond scales, respectively, offering a practical and powerful approach to characterizing beams from accelerators at the frontiers of performance.
\end{abstract}

DOI: 10.1103/PhysRevX.8.021039

Subject Areas: Atomic and Molecular Physics, Plasma Physics

\section{INTRODUCTION}

Advanced particle-accelerator-based scientific-user facilities, such as free electron lasers (FELs) or particle colliders, rely on high-brightness electron beams. X-ray FELs are designed to produce ultrashort and intense bursts of x rays, opening up new research avenues in chemistry, biology, material science, and high-intensity laser-matter interaction physics. Groundbreaking experiments are being performed, ranging from single-molecule imaging to timeresolved ultrafast $\mathrm{x}$-ray science with few-femtoseconds resolution [1-4]. The dynamics of high-gain FELs is directly related to the charge density in space-time and can be expressed by the so-called Pierce parameter, which is optimized by high charge densities, i.e., high peak currents, and few-femtosecond longitudinal and few-micrometer transverse beam sizes [5]. The European X-ray free-electron

* roxana.tarkeshian@iap.unibe.ch

Published by the American Physical Society under the terms of the Creative Commons Attribution 4.0 International license. Further distribution of this work must maintain attribution to the author(s) and the published article's title, journal citation, and DOI. laser (European XFEL), free-electron laser in Hamburg (FLASH), Linac Coherent Light Source (LCLS), SPring-8 Angstrom Compact free electron LAser (SACLA), and Swiss free electron laser (SwissFEL) are examples of such machines that target these extreme parameters with low electron-beam charges of a few picocoulombs and ultrashort pulse durations of a few femtoseconds or shorter as their baseline configurations [6-10]. In future radio-frequency or plasma-based collider concepts, electron beams may be focused to submicrometer transverse beam sizes to maximize the luminosity of the collider, and ultrashort beam durations will help to minimize the amount of beamstrahlung that would impact the performance of accelerators and detectors [11]. The performance of this new generation of facilities also relies on electron beams with high brightness, i.e., high charge density through ultralow emittance. Measuring and, ultimately, controlling the properties of these beams is a major challenge, and novel advanced diagnostic techniques are required to set up and run such accelerators. While the total charge can be readily measured with existing technologies, new concepts are required to access the relevant regimes of few femtosecond to even attosecond beam durations and few micron transverse beam sizes. 
Diagnostic techniques for longitudinal phase-space studies, such as radio-frequency streaking and electro-optic monitoring, have been deployed for few to tens of femtosecond long electron beams [12,13]. Methods relying on either coherent emission in the $\mathrm{THz}[14,15]$ to near infrared and visible regions of the spectrum [16,17] have been successfully used to determine that beams from plasmabased accelerators can be femtoseconds in durations. Recently, a THz-driven streak camera has been proposed with potential femtosecond time resolution, which is achieved by combining resonant $\mathrm{THz}$ subwavelength structures with intense single-cycle $\mathrm{THz}$ pulse generation [18].

Transverse beam sizes are routinely measured, invasively, using scintillator or optical transition radiation screens or wire scanners with resolution of $5-10 \geq \mu \mathrm{m}$, respectively $[19,20]$. Noninvasive laser-based techniques have also been developed to measure the spatial properties of electron beams focused to a few tens of microns [15,21] or even submicrometer scale [22]. However, these techniques, such as the Shintake interference monitor, require dedicated laser systems, as well as precise timing for overlap of femtosecond laser and electron beams.

Lastly, techniques based on the interaction of electron beams and gas have been studied and tested successfully by several authors in order to characterize electron-beam cross sections relying on the space-charge field of the electron beam [23-26].

Here, we revisit ionization-based techniques and propose the use of a high-peak electric field (tens of $\mathrm{GV} / \mathrm{m}$ ) associated with high-brightness electron beams for measuring the charge density on a shot-by-shot basis in a minimally invasive way. Relativistic electron beams with femtosecond or even attosecond durations and transverse beam sizes of micrometers or less carry radially polarized electric fields with tens of $\mathrm{GV} / \mathrm{m}$ field strength. Such fields are sufficiently high to tunnel-ionize a neutral gas through which the electron beam propagates. Detecting and characterizing the yield of ions, their kinetic energy distribution or even their spatial distribution can be used to characterize the electron beam in space and time. The exponential dependency of tunnel ionization on the electron beam's radial space-charge field, and thus its charge density, results in a sensitive monitor for electron beams with longitudinal durations of femtoseconds or even attoseconds and transverse beam sizes on the order of micrometers or less. In principle, the method may have a limited dynamic range in detectable field strength due to the limited number of electrons or ions a detector can measure. However, a significantly larger dynamic range can be obtained by using mixed gas species and/or relying on modification of the kinetic energy spectrum of the ions in a preformed plasma (e.g., created using laser ionization) by the fields of the electron beam below the tunneling ionization field strength. It is important to note that, compared to ionization with laser fields of similar field strengths, ionization with electron beams produces electrons with substantially higher kinetic energies due to the unipolar field. Therefore, it is technically very challenging, if not impossible, to analyze the electrons produced. The proposed diagnostic may serve as a routine tool for optimizing self-amplified spontaneous emission (SASE) FEL operation, and it may also become a key diagnostic for plasma-based accelerators with their ultrashort electron beams [16,17]. It can be used as a complementary technique to the gas monitor detectors (GMD) that are routinely used as a noninvasive and shot-by-shot intensity monitor of FEL photon pulses, relying on photoionization of (rare) gases [27].

In the following sections, we describe the principle of such a charge density monitor and define the operation regimes in which unwanted effects such as competing ionization processes, Coulomb scattering, and plasma shielding can be neglected. The analytical ionization model, complemented by numerical simulations, is summarized. Fundamental differences between electron-beam-based and laser-based tunnel ionization are highlighted, and their influence on the detector performance is discussed. Finally, we analyze to what extent such a diagnostic can be noninvasive.

\section{PRINCIPLE OF THE DIAGNOSTIC METHOD}

The schematic drawing of the charge density diagnostic is shown in Fig. 1. A high-brightness electron beam propagates through a gas plume emanating from a gas jet. A pulsed valve, combined with an appropriate pumping station, ensures good control of the gas pressure, which can be kept within the standard vacuum operating conditions of a linear accelerator $\left(<10^{-6}\right.$ mbar $)$. Detailed knowledge of the density profile in the gas jet is important, including the peak density and length of the gas plume that will be intersected by the particle beam, and can be obtained using interferometric measurement techniques [28]. For sufficiently high space-charge fields, on the order of tens of

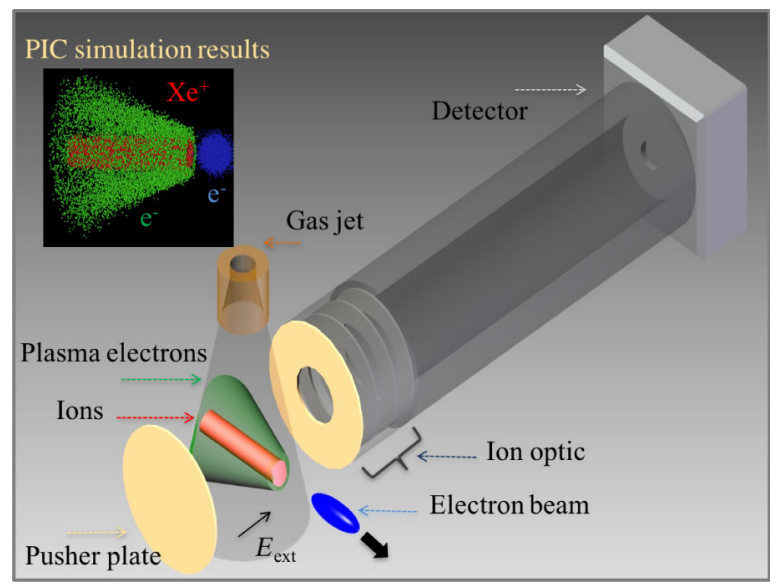

FIG. 1. Schematic of the ionization-based charge density monitor. 
$\mathrm{GV} / \mathrm{m}$, the gas will be ionized by the process of tunneling ionization. The ionized electrons have a few to tens of $\mathrm{keV}$ kinetic energies and will quickly exit the interaction volume, leaving behind an initially static ion column, which subsequently undergoes a Coulomb explosion. A suitable ion detector, e.g., a proportional counter or an ion mass spectrometer, must be designed such that it collects and measures all ions generated. This will become increasingly more challenging as the number of ions becomes larger. In other words, a realistic and accurate simulation of the exploding ion column is essential for a proper understanding of the detector performance. We show that under the proper operating conditions, the number of measured ions is correlated to the peak charge density of the electron beam. In addition, measuring the kinetic energy distributions of the ions can provide information about electron-beam transverse sizes [23,24]. Alternatively, an ion time-of-flight (TOF) spectrometer can be used to retrieve electron-beam parameters through arrival time distributions of multiple species.

\section{THEORETICAL ANALYSIS OF ATOMIC TUNNELING IONIZATION}

We start with an analytical expression for the charge density and the resulting radial electric field distribution, which, for relativistic beams, is polarized predominately in the radial direction. Here, the electric field strength is restricted to a range in which tunneling ionization dominates, e.g., compared to the barrier suppression, and the Ammosov-Delone-Krainov (ADK) model is used to quantify the ionization rate. The charge density distribution can be defined in a cylindrically symmetric geometry as

$$
\rho_{\text {beam }}(r, z, t)=\rho_{\mathrm{pk}} f_{r}(r) f_{z}(z-\beta c t),
$$

with the peak electron charge density $\rho_{\mathrm{pk}}$, the radial distribution $f_{r}(r)$, and the longitudinal distribution $f_{z}(z-\beta c t)$. The radial and longitudinal distributions are normalized such that $\max \left\{f_{r}(r)\right\}=\max \left\{f_{z}(z-\beta c t)\right\}=1$. Moreover, it is assumed that the transverse beam size remains constant over the relevant scale length. The beam propagates along the $z$ axis with a velocity of $\beta c$. Integration over the entire charge density must result in the total beam charge $Q$. The associated radial space-charge field can be readily calculated via Gauss's law,

$$
E_{r}(r, z, t)=\frac{\rho_{\mathrm{pk}}}{\varepsilon_{0} r} \int_{0}^{r} d r^{\prime} r^{\prime} f_{r}\left(r^{\prime}\right) f_{z}(z-\beta c t) .
$$

Hereafter, we illustrate all general results for a Gaussian charge distribution,

$$
\rho_{g}(r, z, t)=\rho_{\mathrm{pk}, g} \exp \left(-\frac{r^{2}}{2 \sigma_{r}^{2}}-\frac{(z-\beta c t)^{2}}{2 \sigma_{z}^{2}}\right),
$$

with the transverse and the longitudinal beam size $\sigma_{r}$ and $\sigma_{z}=\beta c \sigma_{t}$, respectively. In this case, the peak charge density is

$$
\rho_{\mathrm{pk}, g}=\frac{Q}{(2 \pi)^{3 / 2} \sigma_{r}^{2} \sigma_{z}}=\frac{I_{\mathrm{pk}, g}}{2 \pi \sigma_{r}^{2} \beta c},
$$

with the peak current $I_{\mathrm{pk}, g}=Q / \sqrt{2 \pi} \sigma_{t}$. For a Gaussian charge density distribution, the radial electric field is

$$
\begin{aligned}
E_{r, g}(r, z, t)= & \frac{\rho_{\mathrm{pk}, g} \sigma_{r}^{2}}{\varepsilon_{0} r}\left[1-\exp \left(-\frac{r^{2}}{2 \sigma_{r}^{2}}\right)\right] \\
& \times \exp \left(-\frac{(z-\beta c t)^{2}}{2 \sigma_{z}^{2}}\right) .
\end{aligned}
$$

The peak electric field $E_{\mathrm{pk}, g} \approx \rho_{\mathrm{pk}, g} \sigma_{r} / 2 \epsilon_{0}$ occurs at $r \approx \pi \sigma_{r} / 2$.

It would be straightforward to extend this model to describe other current density distributions, but for brevity, this has not been included.

The possibility of creating plasma by tunnel ionizing a neutral gas with the self-field of an intense electron beam was previously studied by several other authors [29-32]. Here, we revisit this work via further analytical modeling and simulations to obtain scaling for the dependency of the generated ions and electrons on the peak charge density of the electron beam.

The ADK model used for this study is based on the ionization rate of a hydrogenlike atom in a static electric field with modifications introduced for many-electron atoms. This tunneling ionization model can be used for the relevant regime of interest for the vast majority of present and near-term future experiments. Nevertheless, it is straightforward to extend the theory and modeling to include ionization by any mechanism. It should also be noted that, if the focused beams were to produce fields in excess of those valid for tunneling ionization, the diagnostic could still be implemented in the tunneling regime by passing the beam some distance outside the gas jet so that the fields within the jet are reduced to achieve ionization in the tunneling regime. In addition, this diagnostic could be used to validate various ionization models by comparing the observed ionization to the results predicted by different models. We envision that beam parameters can be controlled, and hence, levels below and above the critical field could be achieved.

A key element of the ADK theory is that the ionization rate has an exponential dependence on the ionization potential of the atom $\left(V_{i}\right)$ and the strength of the external electric field, which in our case is the radial electric field associated with the high-brightness electron beam. The ionization rate for any type of atom and for a time-varying electric field is given by $[33,34]$ 


$$
\begin{aligned}
W(r, z, t)= & \frac{Z^{2} \omega_{a}}{4 \pi n_{\mathrm{eff}}^{3}}\left(\frac{2 E_{a} Z^{3}}{n_{\mathrm{eff}}^{3} E_{r}(r, z, t)}\right)^{2 n_{\mathrm{eff}}-1}\left(\frac{2 e}{n_{\mathrm{eff}}}\right)^{2 n_{\mathrm{eff}}} \\
& \times \exp \left(-\frac{2 E_{a} Z^{3}}{3 n_{\mathrm{eff}}^{3} E_{r}(r, z, t)}\right),
\end{aligned}
$$

where $\omega_{a}=4.13 \times 10^{16} \mathrm{rad} / \mathrm{s}$ is the atomic unit frequency, $E_{a}=5.1 \mathrm{GV} / \mathrm{cm}$ the atomic unit field strength, $n_{\text {eff }}=Z / \sqrt{V_{i} / V_{H}}$ the effective quantum number, $V_{H}=13.6 \mathrm{eV}$ the ionization potential of hydrogen, and $Z$ the charge of the resulting ion. The ADK formula for hydrogen agrees well with numerical solutions of the time-dependent Schrödinger equation for magnitudes of the external electric field below the critical value $E_{\text {crit }}=$ $E_{a}(\sqrt{2}-1)\left|V_{i} / V_{H}\right|^{3 / 2}[35]$.

All calculations and simulations presented hereafter, unless mentioned otherwise, are performed for typical beam parameters of FEL accelerators: a Gaussian charge density distribution with a total charge of $Q=230 \mathrm{pC}$, a beam duration of $\sigma_{t}=21 \mathrm{fs}$, and a transverse size of $\sigma_{r}=5.67 \mu \mathrm{m}$. These parameters result in a maximum electric field strength of $21 \mathrm{GV} / \mathrm{m}$.

Figure 2(a) shows an intensity plot of the electric field distribution [Eq. (5)] for $t=0$ in the $r z$ plane. The resulting ionization rate for xenon with $V_{i}=12.13 \mathrm{eV}$ is shown in Fig. 2(b). The ionization rate peaks where the radial space-

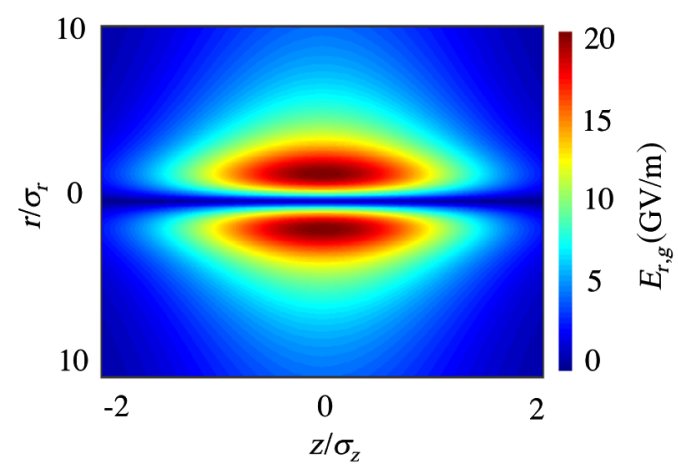

(a)

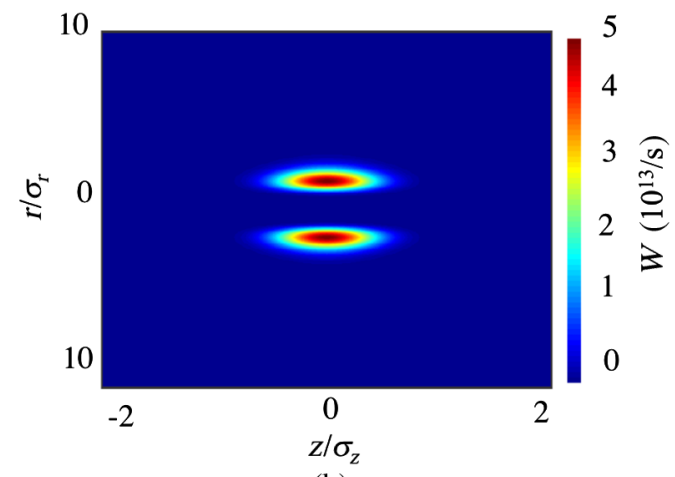

(b)

FIG. 2. (a) Intensity plot of the radial electric field, Eq. (5), in the $r z$ plane and (b) intensity plot of the ionization rate, Eq. (6), in the $r z$ plane. charge field has its maximum. From the ionization rate, the ion density $n(r, z)$ can be estimated, i.e., for $t \rightarrow \infty$,

$n(r, z)=n_{g 0}(r, z)\left[1-\exp \left(-\int_{-\infty}^{\infty} d t W(r, z, t)\right)\right]$,

with the initial gas density $n_{g 0}(r, z)$. The ion density grows linearly with the time-integrated ionization rate if the latter is not too high. While the $r$ dependence of the initial gas density may be neglected for most practical cases, the $z$ dependence has to be considered, as it impacts the total number of ions that can be produced. Moreover, the scale length of the density variation should be comparable to the beta function of the electron beam to assess the length over which ions are generated. The total number of ions generated via field-induced ionization is given by

$$
N_{\text {ions, } \mathrm{ADK}}=\int_{V} d V n(r, z),
$$

where $V$ is the interaction volume of the electron beam with the neutral gas. As an example, the maximum radial electric field and the ionization probability for an electron beam with a peak charge density between 2 and $14 \times 10^{4} \mathrm{C} / \mathrm{m}^{3}$ are plotted in Fig. 3. The neutral gas is xenon with a constant density of $n_{g 0}=10^{17} \mathrm{~cm}^{-3}$. To allow for a consistent comparison of ionization yield for electron beams of various durations, the interaction length is fixed hereafter to $100 \mu \mathrm{m}$, which is less than the beta-function variation along the interaction length for tightly focused (submicron-level) electron beams.

While the peak electric field scales linearly with the peak charge distribution and remains below the critical field for xenon, i.e., $E_{\text {crit }}=63 \mathrm{GV} / \mathrm{m}$, the exponential dependence of the tunnel ionization rate on the electric field results in a highly nonlinear dependency on the total ion number [Eq. (8)] extending over almost 10 orders of magnitude. This makes such an ionization-based monitor, under the

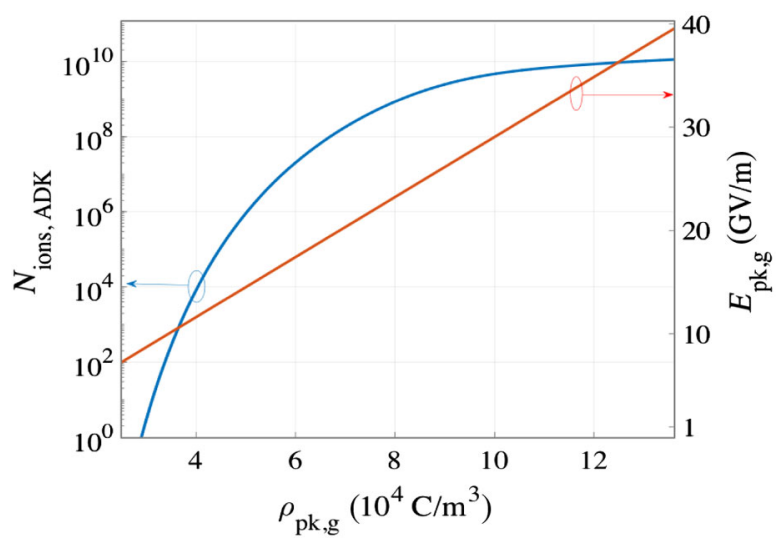

FIG. 3. Peak radial electric field (red curve) and total number of ions (blue curve) versus peak charge density $\rho_{\mathrm{pk}, g}$. 
given conditions, a very sensitive device for the measurement of beam-length changes on a femtosecond or even subfemtosecond scale, assuming that all other parameters, such as gas pressure and transverse beam size, remain constant. By measuring the total number of ions for a known neutral gas density, the peak electric field-and therefore the peak charge density - can be inferred for the (here assumed) Gaussian charge distribution. Since the peak charge density depends on the total charge $Q$, the transverse size $\sigma_{r}$, and beam duration $\sigma_{z}$, by measuring any two of these three parameters via dedicated diagnostics, we obtain the third with unprecedented resolutions. This is especially valuable when one of the three parameters is impossible to measure by any other method.

The rather limited dynamic range in terms of peak electric field values can be easily overcome by installing a focusing magnet before the gas target, which allows one to adjust or even scan the transverse size of the electron beam. In order to have a noninvasive and thus operational diagnostics during user operation, a mixed gas target can be used to extend the dynamic range of the detector in a singleshot basis, as shown in Fig. 4. Here, the ion yield is plotted for four different gases (with all relevant ionization stages) versus electron-beam duration, showing the possibility to extend the range over which electron beams of different durations can be measured.

The inverse problem of determining the longitudinal charge density profile from the measured ion yields does not have a unique solution. For a fixed electron-beam charge and transverse size, there are a variety of charge density profiles that produce the same amount of ions. Owing to the exponential dependence of tunneling ionization on the electric field of the beam, the ionization process will tend to be dominated by any spikes that exist in the charge density profile. Therefore, for example, one cannot distinguish between a smooth Gaussian charge density profile and a profile that consists of a narrow spike on top of a broad background. A short, high-amplitude field could give the same signal as a longer, slightly lower-amplitude

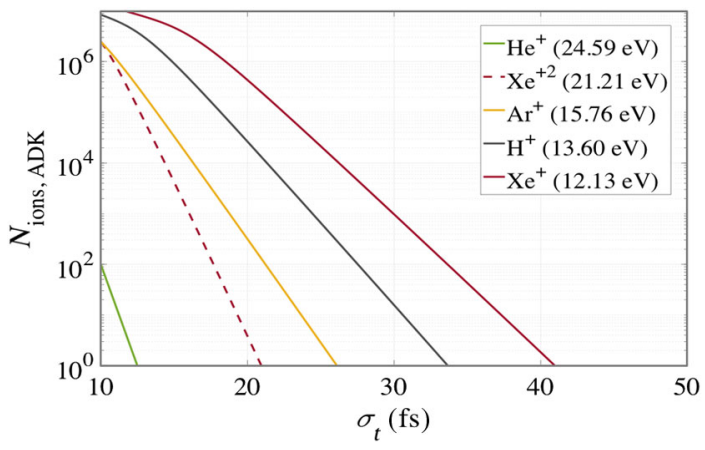

FIG. 4. Total number of ions generated for different gas species (each at $n_{g 0}=10^{14} \mathrm{~cm}^{-3}$ ) versus different electron-beam durations. The electric field can be high enough to double ionize xenon atoms. field. If we assume that the current distribution is smooth, then by fitting the observed signal with a model for a smooth current distribution, one can obtain a lower bound on the maximum current density while realizing that the actual maximum current density may be higher if spikes exist in the current distribution. The proposed technique may aid significantly in measuring and monitoring electron beams on a femtosecond or even shorter timescale. If this diagnostic is combined with another diagnostic that yields information on the width of the spike(s), e.g., coherent transition radiation from a plasma-vacuum boundary (see, for example, Refs. [14,15]), then this limitation could be circumvented. This selectiveness to spikes could well be regarded as being advantageous if the peak current is the parameter of interest - for instance, for the optimization of FELs, where the peak current is one of the decisive parameters for the FEL process.

Time-resolved charge density measurements are crucial for controlling FEL performance. In such cases, the proposed detector may be combined with the emittance spoiling technique proposed by Emma et al. [36]. Alternatively, ultrashort slices could be generated by using a collimator on an energy-dispersed electron beam, if beam losses were not a problem [37]. By using this technique, electron-beam durations on the order of femtoseconds to a few hundred attoseconds (comparable to the FEL slippage length) can be selected and characterized. Scanning the position of the slotted foil allows us to select an unspoiled temporal slice, and by measuring the total number of ions versus foil position, a much more accurate charge density profile measurement may be possible. In addition, the scanning of the slotted foil may be used for a relative calibration of the monitor (number of ions versus beam duration). The intrinsic bandwidth of the SASE process (for most radio-frequency accelerator-driven FELs) would allow selection of unspoiled electron beams of 300 attosecond or more. An important physical effect that could potentially be studied is the quenching of ionization due to nonzero intrinsic quantum tunneling time. This effect may be used as a unique tool to monitor and control attosecond unipolar electron beams that may be produced in FEL machines relying on advanced schemes, such as the enhanced SASE technique [38].

\section{NUMERICAL MODELING OF FIELD- INDUCED TUNNELING IONIZATION}

For a more accurate modeling of the ionization process, numerical simulations were carried out with the fully relativistic particle-in-cell (PIC) codes VSim [39] and WARP [40]. The ADK ionization models used for VSim and WARP can be found in Refs. [41,42], respectively. Electron-beam parameters are those expected for the SwissFEL long-pulse operation mode [10]: a $5.8-\mathrm{GeV}$ electron beam with an energy spread of $\sigma_{E}=350 \mathrm{keV}$, a normalized emittance of $\epsilon_{n}=300 \mathrm{~nm}, 230 \mathrm{pC}$ charge, a 
three-dimensional Gaussian distribution with rms radius of $\sigma_{r}=5.67 \mu \mathrm{m}$, and a rms longitudinal beam duration of $\sigma_{t}=21$ fs. As a neutral gas, we chose xenon, with a density of $\mathrm{n}_{g 0}=17 \mathrm{~cm}^{-3}$ and an interaction length of $100 \mu \mathrm{m}$. These parameters result in a peak charge density of $\rho_{\mathrm{pk}, g}=7.25 \times 10^{4} \mathrm{C} / \mathrm{m}^{-3}$, a peak current of $4.4 \mathrm{kA}$, and an expected maximum radial electric field of $21 \mathrm{GV} / \mathrm{m}$. Figure 5 shows a snapshot of the simulated radial electric field associated with the relativistic electron beam. The lineout indicates a maximum electric field amplitude of approximately $21 \mathrm{GV} / \mathrm{m}$, which is well below the critical field for tunnel ionization of xenon gas but sufficiently high to create a substantial ion density.

Figure 6 shows the theoretical estimation of the peak electric field [Eq. (5), solid magenta curve] as well as the total number of ions [Eq. (8), solid black curve] as a function of the electron-beam duration. Also shown are selected 3D VSim (magenta filled stars) and 2D cylindrical WARP (black diamonds) simulations that agree perfectly well with the analytical predictions. The simulations confirm the highly nonlinear dependence of the total number of ions on the electron-beam duration; i.e., the total number of ions increases from less than 10 at 50 fs to $10^{11}$ for only $10 \mathrm{fs}$. In other words, the measurement features an extremely high sensitivity to small changes of the electron-beam duration.

Even shorter electron beams are expected from laserplasma acceleration (LPA); therefore, the diagnostic performance on the temporal duration of LPA generated electron beams is investigated. To date, the BELLA Laser Facility [43] has generated the highest electron-beam energies of several $\mathrm{GeV}$, and focusing such beams down to few-micrometer spot sizes using novel techniques such as the active plasma lensing [44] is presently being explored.

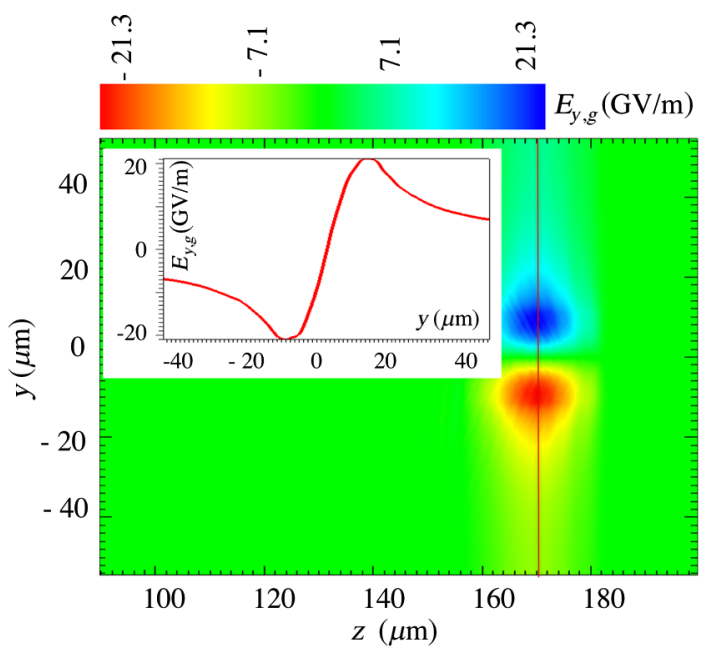

FIG. 5. Radial electric field in the $y z$ plane simulated with the VSim PIC code for a Gaussian charge density with parameters given in the text. The inset graph shows the lineout of the electric field in the radial direction.

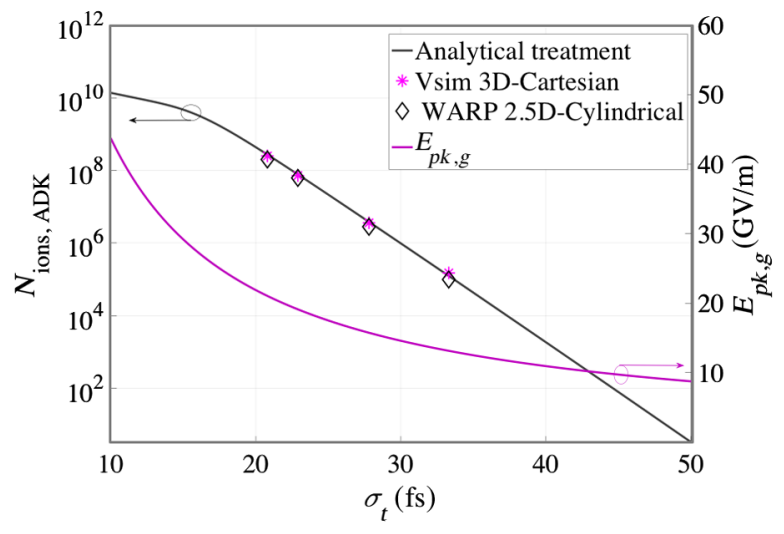

FIG. 6. Total number of xenon ions produced by a threedimensional Gaussian electron beam of various durations. The solid black curve shows the analytical result using the ADK model, the magenta stars are the VSim simulation results $(3 \mathrm{D}$ Cartesian coordinates), and the black diamonds show the results benchmarked by WARP ( $2.5 \mathrm{D}$ cylindrical coordinates). The associated electric field is plotted as a solid magenta curve.

Moreover, the transverse beam size can be readily scanned by changing the discharge current of the plasma lens. The proposed ionization-based monitor could provide information on the electron-beam duration, when the total charge and transverse size are determined independently, and provide an alternative to other electron-beam duration detection schemes $[16,17]$.

Alternatively, if the electron-beam duration is known, the measurement of submicron-size beams with this minimally invasive method is also possible. In the following, the total number of ions generated from BELLA driven electron beams is analyzed theoretically as a function of transverse beam sizes for different electron-beam durations, with the results shown in Fig. 7. The total beam charge is assumed to be $Q=3.3 \mathrm{pC}$ (i.e., at the low side of what is typically produced at BELLA), the argon gas density is $10^{14} \mathrm{~cm}^{-3}$,

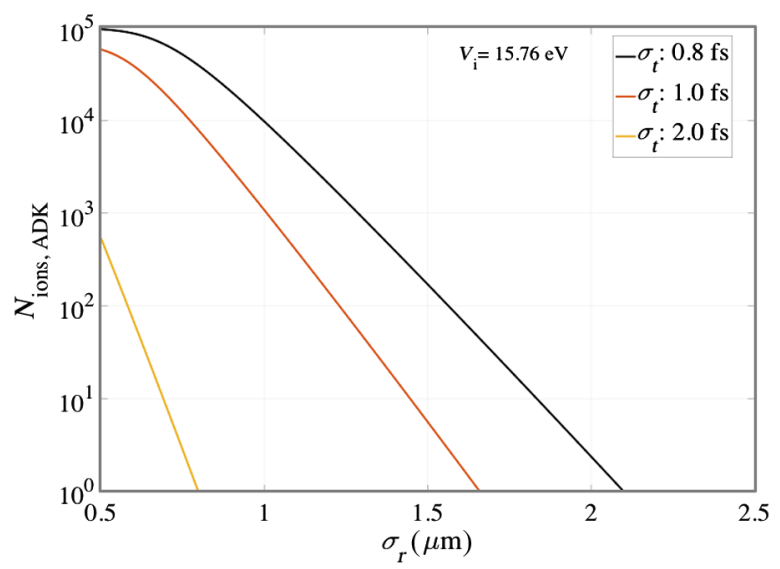

FIG. 7. The number of ions versus transverse beam size $\sigma_{r}$ for different beam durations expected for laser-driven plasma accelerators. 
and the interaction length is $100 \mu \mathrm{m}$. Also, the electric field is kept below the critical field of argon, i.e., $E_{\text {crit }}=$ $93.7 \mathrm{GV} / \mathrm{m}$ for all the cases under study.

The total number of ions depends strongly on the transverse beam size, and only $20 \%$ beam-size variation changes the total number of ions by 1 order of magnitude.

\section{CHARGE DYNAMICS IN NON-NEUTRAL PLASMA}

For a better understanding of the dynamics and detection of plasma electrons and ions, simulations were performed with WARP [40]. Moreover, to incorporate a realistic ion detector, two parallel plates of diameter $D=5.5 \mathrm{~cm}$, thickness $T=5 \mathrm{~mm}$, and separation $S=1.5 \mathrm{~cm}$ were included. The bottom plate has a potential of $V=600 \mathrm{~V}$ to push the ions towards the grounded top plate, with an aperture of diameter $A=1 \mathrm{~cm}$ at the center. The aperture simulates the entrance to, e.g., an ion time-of-flight spectrometer. As a gas target, hydrogen or xenon was selected, and the density was varied between $10^{11} \mathrm{~cm}^{-3}$ and $10^{17} \mathrm{~cm}^{-3}$. The gas plume was approximated by a $100 \mu \mathrm{m}$ flat density section terminated at both ends by a $10 \mu \mathrm{m}$ sinusoidal ramp. To capture the wide range of length scales accurately, WARP's adaptive mesh refinement was used. A nested set of grids of decreasing size and increasing resolution (by factors of 2 along each direction at each refinement level) was adaptively determined for each iteration step, enabling field solutions of the ion column and plates together. The entire grid spanned around 3 orders of magnitude in each dimension using only 40960 cells instead of the over $1.3 \times 10^{9}$ that would be required for a fixed grid. Also, adaptive time stepping was used to speed up the simulations.

First, analytical scaling expressions are discussed to show the response of plasma electrons and ions to the electron beam's space-charge field with and without the aid of an external extraction field. Thereafter, the plasma dynamics of laser-induced and electron-beam-induced ionization is compared.

\section{A. Plasma effects and Coulomb explosion}

For a preionized, uniform plasma, the plasma response to a relativistic electron beam is studied in detail in Refs. [29,45]. For a low-density plasma driven by a short, intense electron beam (in which the plasma wavelength is long compared to the beam length and the plasma density is lower than the beam density), the plasma develops a plasma wake (electron plasma wave) in the blow-out or bubble regime $[46,47]$.

Here, the plasma electrons are forced outwards radially by the Coulomb field of the electron beam, leaving behind the ions, which are essentially stationary on the timescale of a plasma period. For an initially uniform plasma (of infinite radial extent), the plasma electrons are blown out to some maximum radius (the bubble radius), before they are pulled back to the axis by the field of the ions within the bubble region. For an initially uniform plasma, the electrons will always return to the axis, even when increasing the electron beam's field, which will force the plasma electrons outwards to larger radii; the unshielded ion region within the bubble, and thus the restoring force, increases concurrently, thereby causing the electrons to return to the axis.

For a plasma of finite radius, this is not necessarily the case. If the plasma radius is larger than the bubble radius, we expect the bubble structure to be largely unperturbed from the uniform plasma case. If the plasma radius is smaller than the bubble radius, then the restoring force is reduced since the number of ions within the bubble is reduced (owing to the finite radial extent of the plasma). This causes the maximum radial extent of the blown-out electron orbits to increase. For sufficiently narrow and lowdensity plasmas, the restoring force of the ions cannot balance the radial force of the drive beam, and the electrons will not return to the axis but instead will radially escape the plasma. Once the electrons escape, an unshielded ion column will remain, which will subsequently undergo a Coulomb explosion with energy gain proportional to the space-charge field of the ion column.

The electrons can escape when the energy gained from the electron beam is greater than the potential of the ion column, which will be satisfied for $n_{b} / n_{0} \gg Z_{i} e r_{p}^{2} /\left(L_{b} r_{b}\right)$, where $Z_{i} e$ is the ion charge, $L_{b}$ and $r_{b}$ are the beam length and radius, respectively, $n_{b}$ and $n_{0}$ are the densities of the beam and ion column, respectively, and $r_{p}$ is the radial size of the plasma column. This condition is well satisfied for all scenarios presented here.

Estimates and scaling laws for ion energies resulting from Coulomb explosion can be obtained by considering a simplified model of a uniform plasma column of finite radius. If we consider a long ion column with initially uniform ion density $n_{0}$ out to a radius $r<r_{p}$, the kinetic energy gained by an ion after moving from $r_{i}<$ $r_{p}$ to $r_{f}$ is $\mathcal{E}_{\mathrm{C}}=M_{i} c^{2}\left(k_{i} r_{i}\right)^{2} \ln \left(r_{f} / r_{i}\right) / 2$, where $k_{i}=$ $\sqrt{n_{0} Z_{i}^{2} e^{2} / \epsilon_{0} M_{i} c^{2}}$ is the ion plasma wave number and $M_{i}$ the ion mass.

Figure 8 shows the maximum energy gain of ions, e.g., hydrogen and xenon ions, owing to the Coulomb explosion, versus gas density. For low gas densities, the total number of ions is small, and the kinetic energy gain after Coulomb explosion is smaller than the energy gained in the external extraction field. For gas densities higher than approximately $8 \times 10^{15} \mathrm{~cm}^{-3}$ (for hydrogen), the situation reverses and the kinetic energy gain is dominated by the Coulomb explosion.

Collective effects in the ion column can dominate the ion motion compared to the effects of the external extraction field. This is the case when the space-charge field in the ion column is greater than the applied field. As the ion column expands via the Coulomb explosion, the space-charge field 


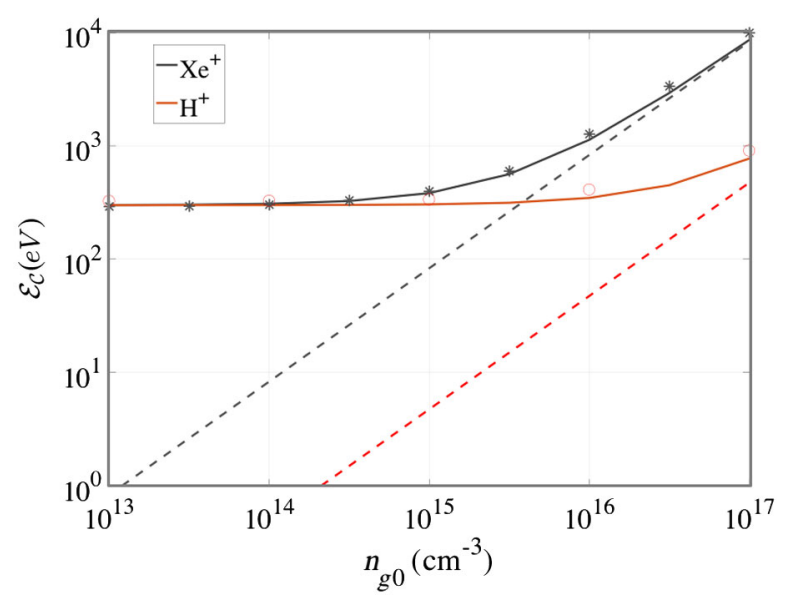

FIG. 8. Kinetic energy gain of hydrogen (red curve) and xenon ions (black curve) in the presence of Coulomb and external extraction field versus gas density. The dashed curves show the energy gained because of Coulomb explosion alone.

is reduced and the external extraction field will start to impact the ion trajectories. In order to estimate the size of the ion space-charge field during expansion, consider an ion column with initially uniform ion density $n_{0}$ out to a radius $r<r_{p}$. At a radius of $r_{f}$, the field is given by $E=n_{0} Z_{i} e r_{p}^{2} / 4 \epsilon_{0} r_{f}$. When the ion space-charge field is much less than the external extraction field, the latter one dominates the ion motion. A relatively high gas density of $10^{17} \mathrm{~cm}^{-3}$ or more results in a relatively high ionic Coulomb field, as shown in Fig. 9. The field-induced ions are enhanced at the surface of the electron beam, where the radial electric field of the electron beam has its maximum (Fig. 9). The space-charge field, for the case where a large amount of ions is produced in a small volume, can reach hundreds of $\mathrm{MV} / \mathrm{m}$.

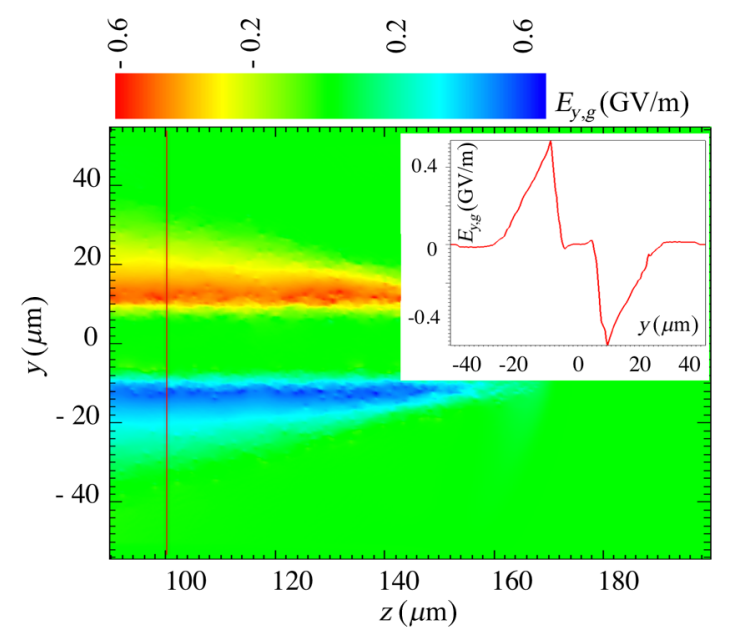

FIG. 9. VSim PIC simulation reveals the electric field resulting from the fast electrons leaving the ions behind unshielded. The inset graph shows the lineout of the plasma field in the transverse direction.
To have an ion space-charge field less than a typical external extraction field of approximately $40 \mathrm{kV} / \mathrm{m}$ at a high density $\left(10^{17} \mathrm{~cm}^{-3}\right)$ would require an expansion of the ion column by 4 orders of magnitude, i.e., from $5 \mu \mathrm{m}$ to $5 \mathrm{~cm}$. In the low-density case, e.g., for $10^{12} \mathrm{~cm}^{-3}$ or lower, expansion by 2 orders of magnitude would be sufficient for the external extraction field to dominate. Note that, although both electrons and ions can reach a typical TOF detector, because of the large velocity difference, they will be well separated in time.

As was discussed above, in the low-density regime, where Coulomb effects are minimized, electron-beam properties may be inferred by simply monitoring the plasma density (ion numbers). But even in the high-density regime, a measure of the electron-beam transverse size can be obtained from the arrival time of the ions. In a nonneutral plasma, the large ion kinetic energies due to the

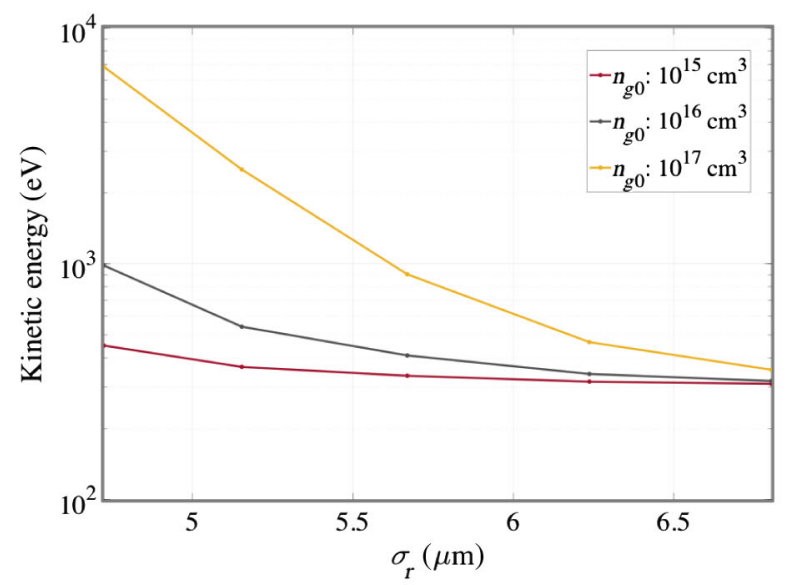

(a)

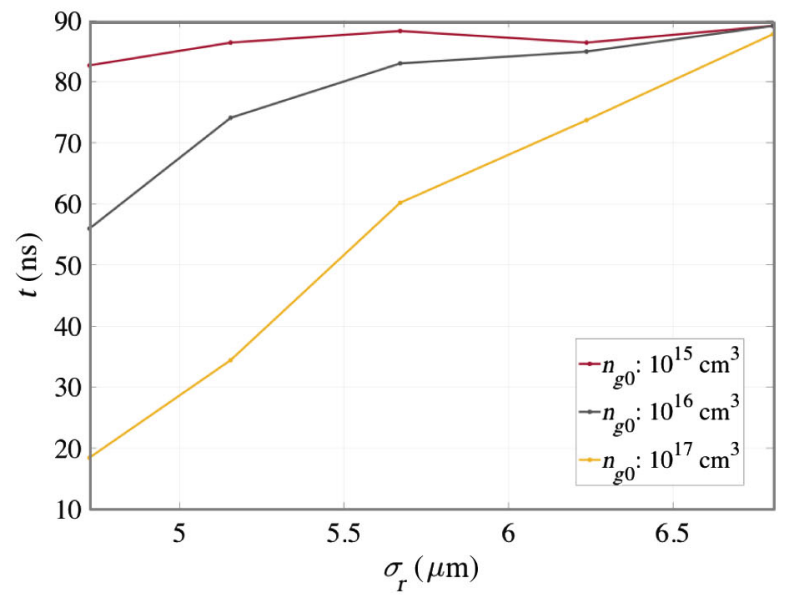

(b)

FIG. 10. (a) Kinetic energy imparted on protons due to the Coulomb forces in the non-neutral plasma for electron beams with different transverse size and different initial neutral gas densities. (b) Arrival time of protons in the detector for different neutral gas densities. Results are obtained with the WARP simulation code. 
Coulomb explosion vary linearly with the ion numbers and exponentially with the space-charge field of the electron beam. Figures 10(a) and 10(b) show the kinetic energy and associated arrival time of protons onto the detector, respectively, as a function of electron-beam transverse size and for different neutral gas density.

The energy and arrival times are directly related to the Coulomb forces in the non-neutral plasma, i.e., after the plasma electrons have already escaped. As can be seen, beam-size changes from 5 to 5.5 microns result in arrival time differences of a few tens of nanoseconds, enabling ultrahigh (submicron) precision. The plasma dynamics after the ionization process and the tailoring of the detection scheme allows us to measure different electron-beam properties.

In the next section, the plasma temperature induced by a laser is compared to the one induced by an electron beam. The simulations will show that an electron beam, with a unipolar space-charge field, produces electrons with a much higher average kinetic energy than what is commonly expected from a laser beam. In fact, their kinetic energy turns out to be so high that a diagnostic based on electron detection becomes extremely challenging to realize. For that reason, and also because an electron detection scheme would not allow us to differentiate between ionization from different gas species, the remainder of this section focuses on ions, i.e., their dynamics after ionization. Specifically, we address the question of ion detection efficiency, that is, the ratio of detected over generated ions.

\section{B. Electron- versus laser beam-induced tunnel ionization}

The electron-beam parameters are identical to the ones used above, and the laser beam intensity was adjusted to get a similar total number of ions. The laser beam $(\lambda=800 \mathrm{~nm})$ with duration of $\sigma_{t}=6.7 \mathrm{fs}$, focused to $2 \mu \mathrm{m}$, provides an electric field strength of $E_{0}=65 \mathrm{GV} / \mathrm{m}$ with an intensity of $5.5 \times 10^{14} \mathrm{~W} / \mathrm{cm}^{2}$, a normalized vector potential of $\mathrm{a}_{0}=0.016$, and hence low temperature of the initial plasma.

Figure 11 shows the field-induced ionization of xenon gas with a density of $10^{17} \mathrm{~cm}^{-3}$. The two snapshots [Fig. 11(a) for the electron and Fig. 11(b) for the laser beam] are taken shortly after the electron or laser beam (blue area) has passed the interaction region. While ions (red dots) are almost stationary in both scenarios, electron dynamics (green dots) differ strongly. This can be seen more quantitatively when analyzing the kinetic energy distribution of electrons and ions. The average ion kinetic energy is less than an $\mathrm{eV}$, i.e., $99.5 \mathrm{meV}$ for the electron beam [Fig. 11(e)] and $14.1 \mu \mathrm{eV}$ for laser beam ionization [Fig. 11(f)]. Inspecting the plasma electron kinetic energy distribution for the two cases reveals a different picture; see Figs. 11(e) and 11(f). Electron beams generate much higher kinetic energies compared to laser beams. Here, we find an average kinetic energy of $23.2 \mathrm{keV}$ for electron beams and
Electron beam induced tunnel ionization

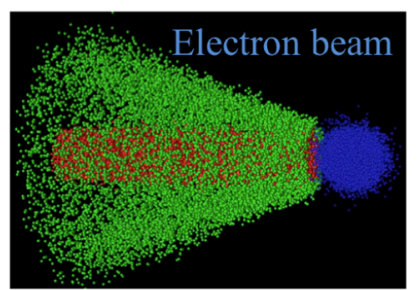

(a)
Laser beam induced tunnel ionization

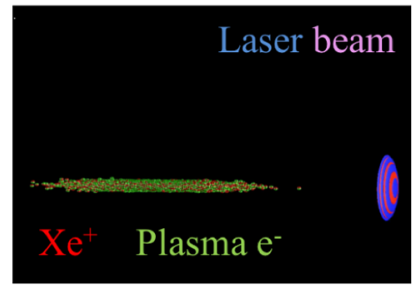

(b)

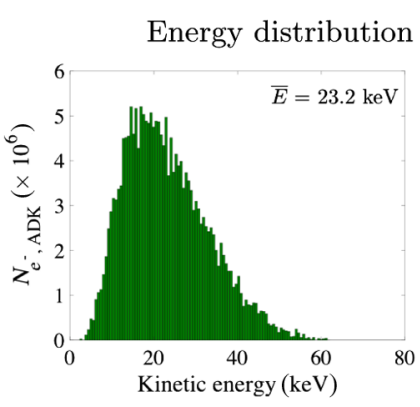

(c)

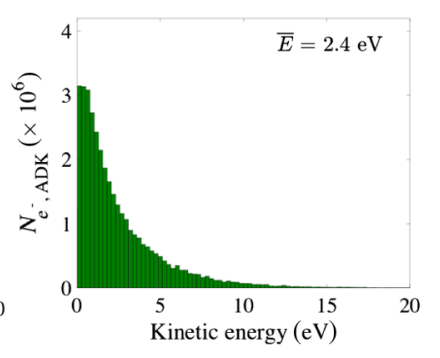

(d)
Energy distribution of xenon ions

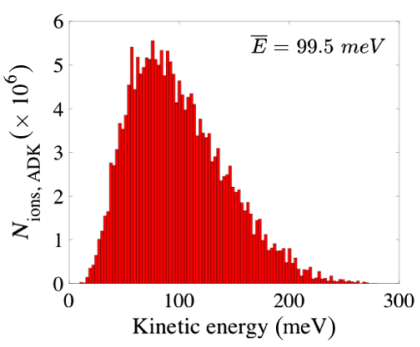

(e)

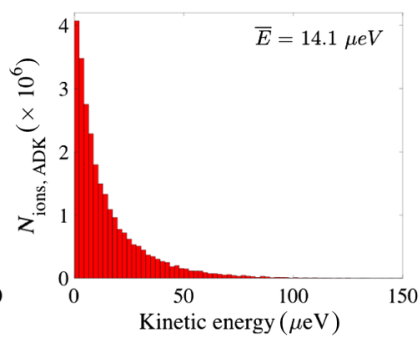

(f)
FIG. 11. Three-dimensional distribution of electrons (green dots) and ions (red dots) after the relativistic electron beam has passed the $100 \mu \mathrm{m}$ gas plume; kinetic energy distributions of plasma electrons and xenon ions are shown in panels (c) and (e), respectively. The corresponding simulation results for a laser beam are shown in panels (b), (d), and (f). The results are taken from WARP particle-in-cell code, benchmarked by VSim.

$2.4 \mathrm{eV}$ for laser beams. The dramatic difference is related to the unipolar nature of the radial Coulomb field associated with electron beams. While the electromagnetic field of lasers wiggles electrons on an oscillatory trajectory, the unipolar field of electron beams accelerates plasma electrons outward radially.

Thus, electron-beam tunnel ionization is fundamentally different from laser-induced tunnel ionization or x-ray photoionization with $a_{0} \ll 1$ [48-50], where the electrons are created with very small initial drift velocities and hence remain close to the ions for an extended time [51-53]. The relatively cold electrons separate from the ions because of the external extraction electric field. Therefore, electrons, as well as ions, can be analyzed in order to gain information about the ionization process. Electrons are analyzed, for example, in streaking experiments with IR or $\mathrm{THz}$ 
radiation, where the electron kinetic energy distribution provides information on the duration of the ionization pulse and its arrival time [54-56]. Conversely, intense unipolar fields of electron beams impart a significant momentum (multi-keV/c) to the plasma electrons, which makes it difficult to capture them, except for (unrealistically) high extraction fields. Similarly to the photo-induced ionization diagnostics, when combined with a THz streaking field, the electron-beam's duration as well as its arrival time may be retrieved from plasma electrons at the few-keV/c level. The process of capture and detection of the generated ions, which have lower kinetic energies, is more favorable for direct measurement of electron-beam properties and hence will be discussed hereafter.

Whether the method relies on the total number of detected ions or their kinetic energy, evaluation of the detector's collection efficiency is essential and is discussed further in the following section.

\section{CHARGE COLLECTION EFFICIENCY}

The detector performance and its collection efficiency depend, to a large extent, on the gas density and ionization level. In a regime where either the initial gas density or the ionization probability is low, only a small number of ions are generated (e.g., up to a few hundred ions). In this scenario, especially for high-mass ions, the ion behavior can be well described using single-particle equations where the external extraction field dominates the ion dynamics. On the other hand, for low-mass ions or protons, the effect of the external extraction electric field is of the same order of the initial radial kick from the initial electron beam, while for electrons, the initial radial kick dominates. The corresponding drift time is given by $\Delta t_{D}=$ $S \sqrt{M_{i} /\left(Z_{i} e\right) \Phi}$, with extraction field $\Phi$, ion charge $Z_{i} e$, ion mass $M_{i}$, and deflection plate separation $S$. For lowmass ions or protons, the radial field of the electron beam of duration $\sigma_{t}$ may have to be considered, which would result in a transit time of $\Delta t_{R} \approx S M_{i} /\left(2 Z_{i} e E_{r} \sigma_{t}\right)$. The ratio of $\Delta t_{R} / \Delta t_{D}$ scales as $\sqrt{m}$ and can be used to estimate the required extraction field for efficient ion detection.

As an example, assume an electron beam with duration $\sigma_{t}=21 \mathrm{fs}$, radial electric field $E_{r}=21 \mathrm{GV} / \mathrm{m}$, and deflection plates separated by $S=1.5 \mathrm{~cm}$, with an extraction voltage of $\Phi=600 \mathrm{~V}$. The radial expansion times $\Delta t_{R}$ are approximately $97 \mathrm{ps}, 178 \mathrm{~ns}$, and $23 \mu \mathrm{s}$ for electrons, protons, and xenon ions, respectively, while the corresponding drift times $\Delta t_{D}$ are approximately $1.5 \mathrm{~ns}, 63 \mathrm{~ns}$, and $717 \mathrm{~ns}$. Hence, in this case, radial expansion is the dominant effect for electrons, which barely have time to drift, while the timescales for expansion and drift are of the same order for protons, and the drift is the dominant effect for the high-mass xenon.

Figure 12(a) shows the total ion charge generated (black solid curve) and also the total ion charge (red dashed curve)

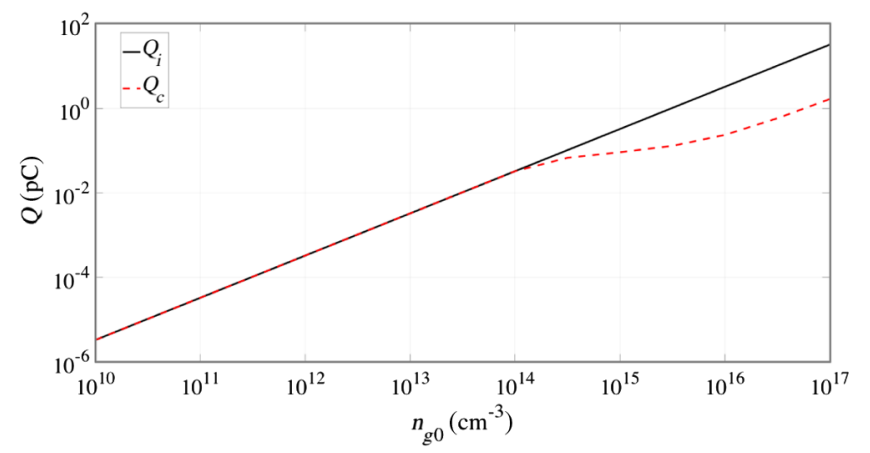

(a)

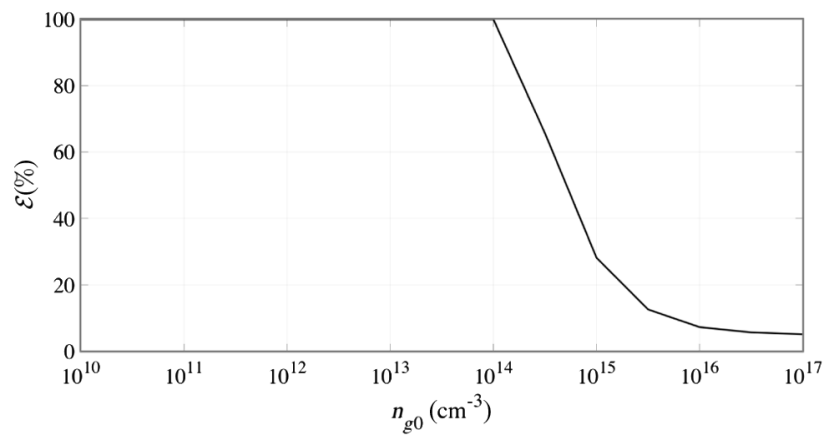

(b)

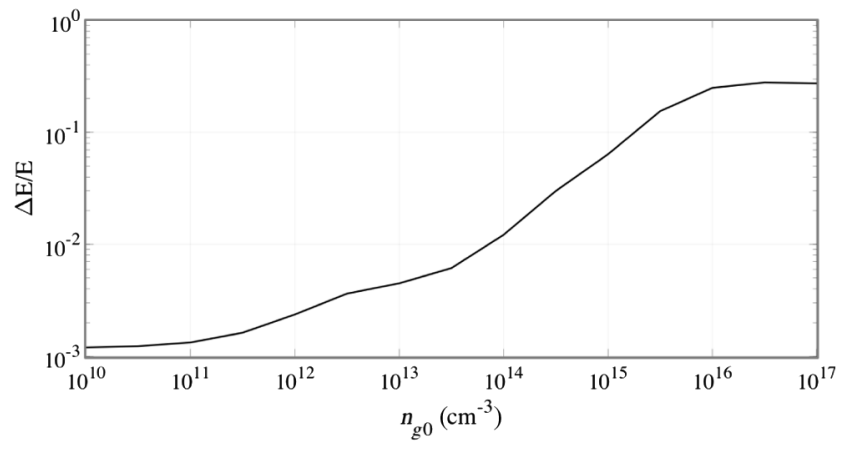

(c)

FIG. 12. (a) Total number of field-induced ions (black solid curve) and detected (red dashed curve) versus xenon gas density. (b) Collection efficiency for a TOF detector with $1 \mathrm{~cm}$ aperture size and $600 \mathrm{~V}$ external voltage. (c) Energy spread of xenon ions passing through the aperture in the top deflection plate.

passing through the aperture in the top deflection plate. The associated collection efficiency is shown in Fig. 12(b). In order to have a reliable measurement, the total ion charge should be kept below $100 \mathrm{fC}$, which, for the parameters used here, is reached for a maximum xenon gas density of $10^{14} \mathrm{~cm}^{-3}$. If the ion detector is a time-of-flight spectrometer, it is important that the relative energy spread of the ions entering the spectrometer does not exceed $10^{-2}$ in order to increase mass selectivity. Figure 12(c) indicates that this is the case as long as the gas density is below $10^{14} \mathrm{~cm}^{-3}$, which coincides with the threshold density obtained above.

Next, the ion dynamics for mixtures of different gases, here xenon (as a high- $Z$ element) and hydrogen (as a low- $Z$ 

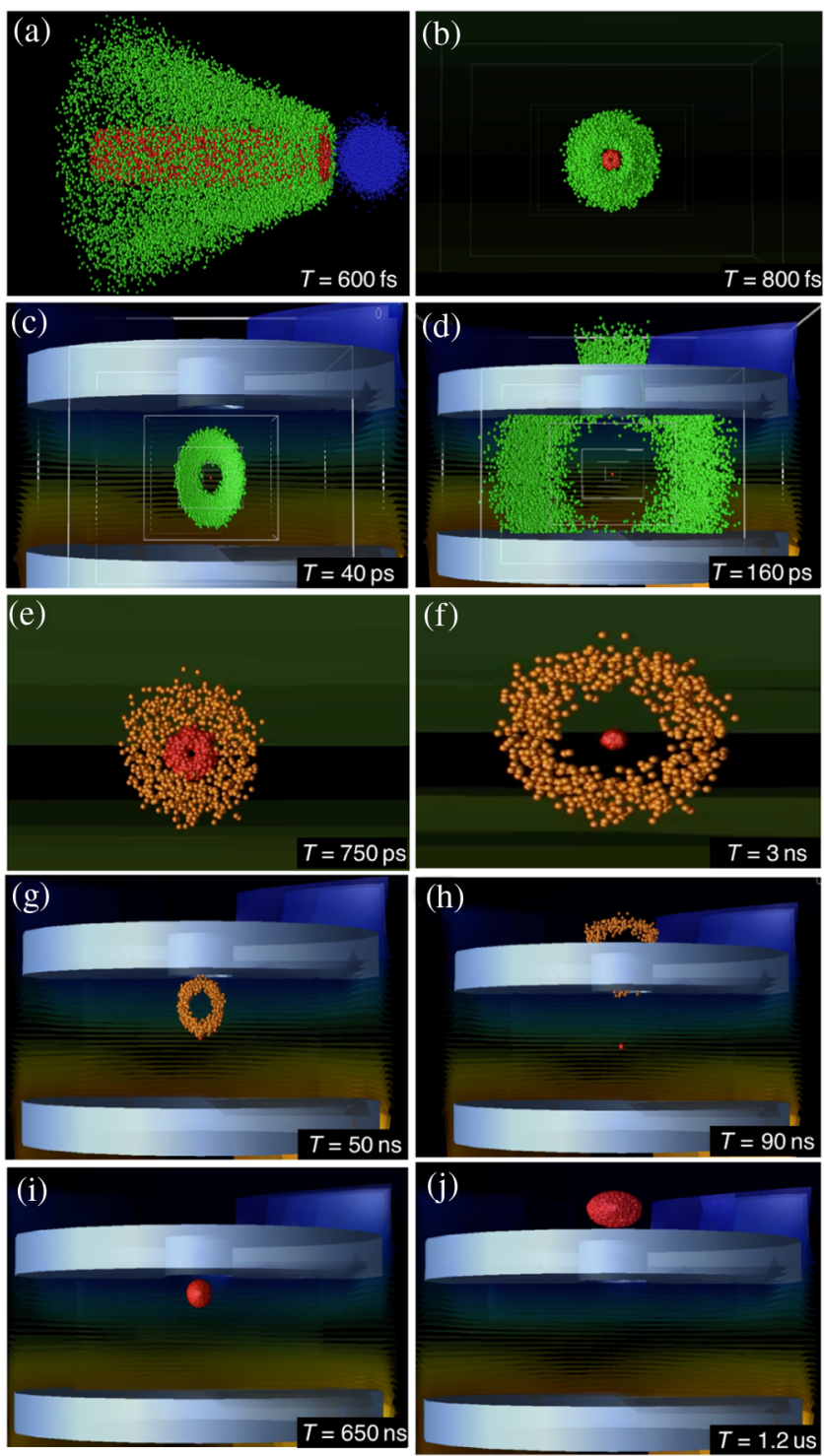

FIG. 13. Time-ordered sequence of snapshots showing electrons (green dots), protons (orange dots), and xenon ions (red dots) being expelled from the interaction volume. Simulations were done with WARP. (a) An electron beam (blue circles) passes through a mixture of hydrogen and xenon gas. The plasma electrons, with their high kinetic energy, rapidly leave the ion assembly. Afterwards, the ions undergo Coulomb explosion, first the lighter hydrogen and later the heavier xenon ions. The external extraction field is sufficiently high $(40 \mathrm{kV} / \mathrm{m})$ to move both ion species through the aperture in the top plate before they expand to a diameter larger than the aperture size.

element), both at a density of $10^{14} \mathrm{~cm}^{-3}$, are analyzed. Gas mixtures, as discussed above, may result in a larger dynamic range of the charge density monitor. Figure 13 shows the evolution of the plasma upon ionization by the electron beam on different timescales and length scales.

Figures 13(a) and 13(b) display a three-dimensional side and front view of the rapidly expanding electrons and the initially stationary ion column just after the electron beam leaves the interaction volume. The extraction field cannot compete with the radial Coulomb field of the electron beam, and as a consequence, the electron distribution expands very rapidly and only a small fraction of electrons passes through the aperture [Figs. 13(c) and 13(d)]. After the electrons have left, the ion column starts to undergo Coulomb explosion. The first ions to explode radially are the much lighter protons [Figs. 13(e)-13(g)]. Here, the extraction field is just high enough to transport all protons through the top plate's aperture [Fig. 13(h)]. Lastly, the much heavier xenon ions start to expand. Their radially outward directed expansion is almost negligible compared to the collective upward motion driven by the extraction field [Figs. 13(j) and 13(k)]. Note that the dynamics illustrated in Fig. 13 describes the ideal situation where the number of detected ions (the ones passing through the aperture) is equal to the number of generated ions.

The total ion charge for xenon and hydrogen is $18 \mathrm{fC}$ and $1 \mathrm{fC}$, respectively, which is in good agreement with theoretical predictions and within the dynamic range of the detectors. As was discussed above, the arrival time of the different species to the ion detector has a $\left(M_{i} / Z_{i} e\right)^{1 / 2}$ dependency; therefore, protons arrive first, followed by xenon ions. It is interesting to note that the initial ring shape of the radial proton distribution is maintained until the top plate is reached; see Fig. 13(g). Such behavior is expected whenever the extraction field dominates the Coulomb explosion dynamics, i.e., under normal operation conditions of the charge density monitor. This behavior becomes even more obvious when we plot the minimal and maximal radii of the ion distribution as a function of time, as shown in Fig. 14.

The ring shape of the hydrogen distribution is maintained over 100 nanoseconds. Moreover, the arrival time difference between the innermost and outermost ions is

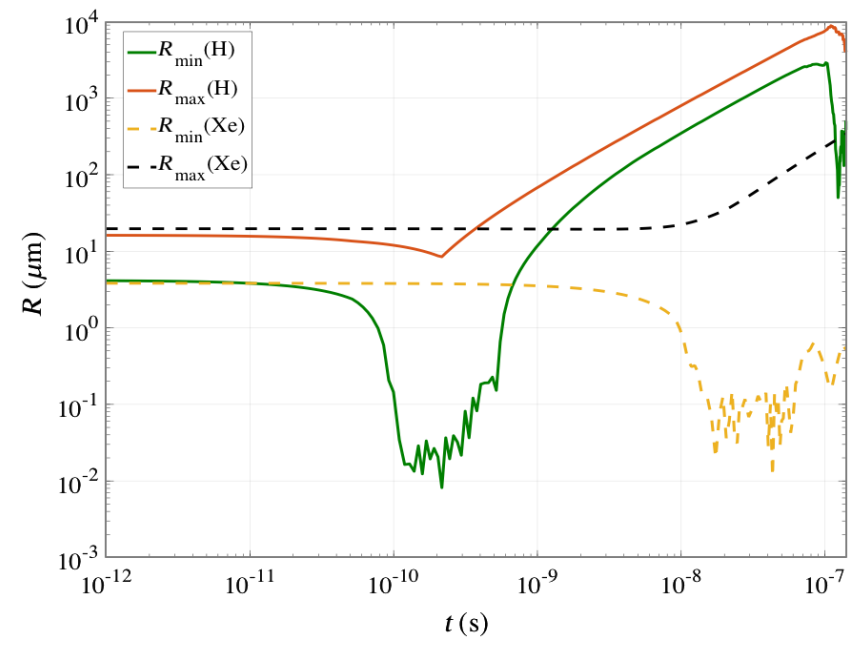

FIG. 14. Time evolution of the inner radius $R_{\min }$ (green solid curve for hydrogen and yellow dashed curve for xenon) and outer radius $R_{\max }$ (red solid curve for hydrogen and black dashed curve for xenon) of hydrogen and xenon distribution. 

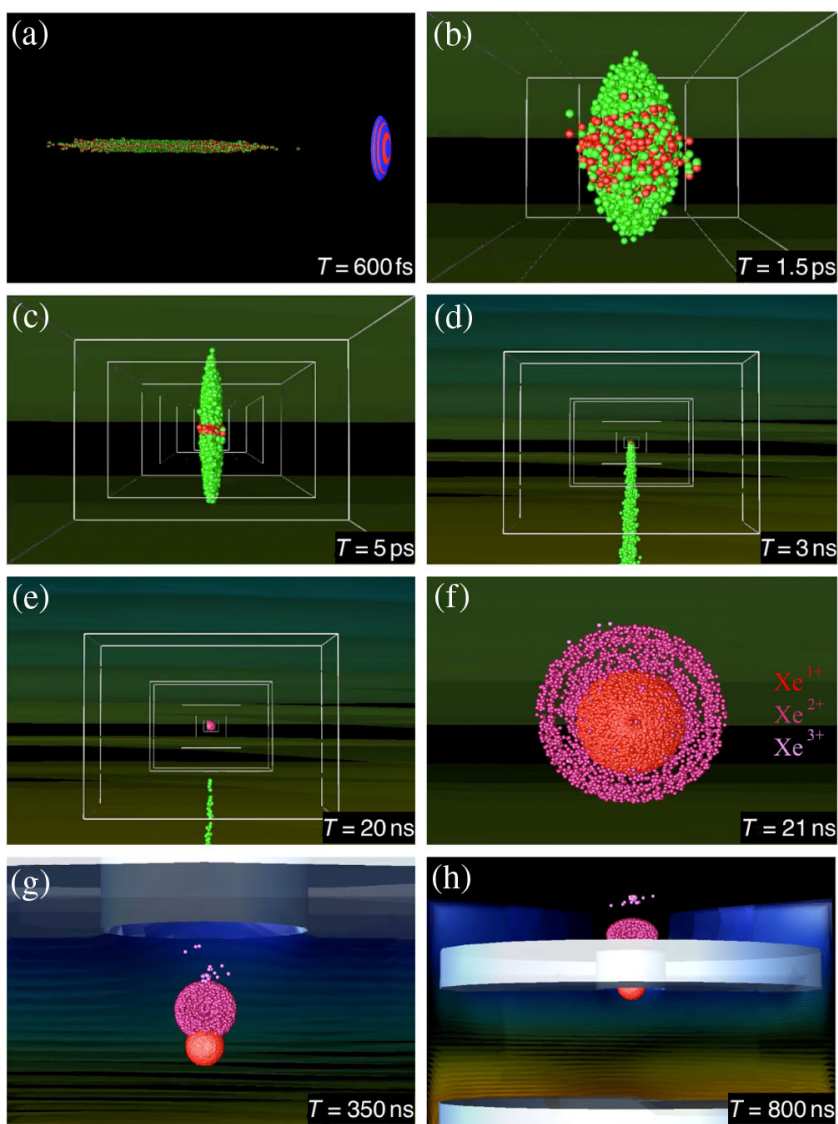

FIG. 15. Time-ordered sequence of snapshots showing the dynamics of electrons (green dots), $\mathrm{Xe}^{+}$(red dots), $\mathrm{Xe}^{2+}$ (pink dots), and $\mathrm{Xe}^{3+}$ ions (light pink dots). Simulations were done with WARP. (a) A laser beam (blue oval) passes through a xenon gas. The cold plasma electrons expand but move collectively to the bottom plate. Simultaneously, the external field pulls the xenon ions through the aperture in the top plate before they expand to a diameter larger than the aperture size.

approximately $30 \mathrm{~ns}$. This would allow us to retrieve electron beam transverse size via monitoring the arrival time distribution of the protons.

Lastly, we show the corresponding dynamics of a laserinduced plasma. Figure 15 shows a time-ordered sequence of snapshots of the electron and ion dynamics. The laser's intensity is high enough to produce $\mathrm{Xe}^{+}$(red dots), $\mathrm{Xe}^{2+}$ (pink dots), and $\mathrm{Xe}^{3+}$ ions (light pink dots). Depending on their charge, they pass the aperture in the top plate in successive order. As stated above, and contrary to electronbeam ionization, electrons and ions from photo or laser ionization can be used for diagnostic purposes.

\section{BACKGROUND DETECTOR SIGNALS DUE TO OTHER IONIZATION EFFECTS}

There are several competing ionization mechanisms that contribute to the total ion yield which are not included in the PIC simulations. One such mechanism is impact ionization by the relativistic electron beam itself as well as by the plasma electrons. Another mechanism, which is only relevant in laser- or beam-driven plasma acceleration schemes, is photoionization via betatron radiation [57-63]. The number of background ions produced by these competing ionization processes can be estimated as

$$
N_{\text {ions,bkg }}=n_{g 0} \int_{V} d V \sum_{j} \int_{0}^{\infty} d E \sigma_{j}(E) f_{j}(E),
$$

where $n_{g 0}$ is the gas density, $j$ indicates a specific ionization process, and $\sigma_{j}(E)$ is the corresponding energy-dependent ionization cross section. The cross section for impact ionization of xenon by relativistic electron beams in the energy range of $10 \mathrm{eV}-100 \mathrm{GeV}$ can be found in Ref. [64].

Impact ionization from plasma electrons was assessed for the case of a beam generating a relatively high field of $21 \mathrm{GV} / \mathrm{m}$, with a peak current of $4 \mathrm{kA}$ and total charge of $230 \mathrm{pC}$. The spectrum of plasma electrons [see Fig. 11(c)] ranges from a few to $60 \mathrm{keV}$ kinetic energy. Operating at a low gas density of $10^{13} \mathrm{~cm}^{-3}$ and a gas jet length of order $100 \mu \mathrm{m}$ will result in a relatively low number of plasma electrons and ions $\left(10^{4}\right)$, which, in turn, will be insufficient to generate a detectable number of additional impact ionization events over the radial interaction length.

In the case of betatron emission in laser plasma accelerators, photons with energies in the range of $\mathrm{keV}$ to few tens of $\mathrm{keV}$ are generated. These photons copropagate with the electron beam, with a typical beam divergence of $1 / \gamma$. For high-energy electron beams, this angle can be smaller than the electron-beam divergence without the use of focusing or collimating elements. The photoionization cross section for xenon in the vacuum ultraviolet (VUV) and x-ray regime is tabulated in Ref. [65].

Figure 16 compares the total number of xenon ions due to the different contributions as a function of the total beam charge.

The black, orange, and yellow curves depict the total number of ions produced via tunnel ionization for different parameters, i.e., for $\sigma_{z}, \sigma_{r}=1 \mu \mathrm{m}, 3 \mu \mathrm{m}$, and $5 \mu \mathrm{m}$. The violet and green curves show the total number of ions produced via impact ionization by the electron beam (violet curve) and photoionization by betatron radiation (green curve). Figure 16 illustrates that competing ionization mechanisms are non-negligible for low charge cases when the radial space-charge field is below the threshold needed for tunnel ionization.

Minimizing the contribution of impact ionization for particular experimental conditions therefore has to be achieved empirically through tuning of the gas density and gas jet scale length, as well as with the minimum required electron beam charge for high-brightness electron beams. 


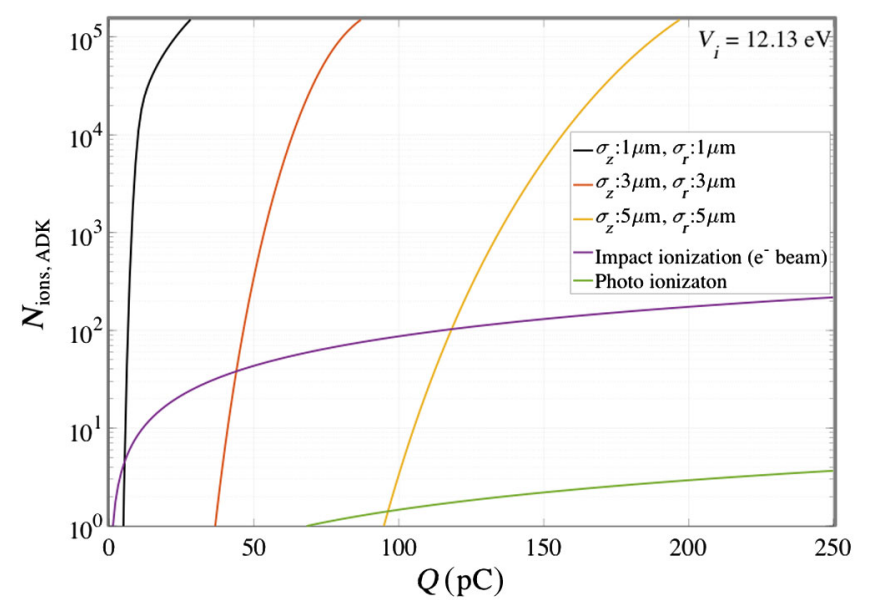

FIG. 16. Comparison of different ionization mechanisms in xenon gas. The plot shows the total number of ions as a function of the electron bunch charge for different electron-beam parameters and ionization mechanisms. Tunnel ionization by an electron beam with $\sigma_{z}=\sigma_{r}=1 \mu \mathrm{m}$ (black curve), $\sigma_{z}=\sigma_{r}=$ $3 \mu \mathrm{m}$ (orange curve), and $\sigma_{z}=\sigma_{r}=5 \mu \mathrm{m}$ (yellow curve). We show impact ionization by the electron beam (violet curve) and photoionization due to betatron radiation (green curve).

\section{IONIZATION-BASED TECHNIQUE AS A NONINVASIVE METHOD}

To evaluate whether the concept is largely noninvasive, we studied the emittance degradation and the possibility of the generation of plasma wakefields that would act on the main drive beam. An emittance increase due to multiple scattering events by the electron beam traveling through a gas and plasma mixture was estimated and found to be negligible for typical experimental conditions of relatively low-density short gas targets. In addition, emittance diffusion can occur through multiple scattering from the ion channel and, predominantly, from neutral gas atoms. The normalized rms emittance growth due to multiple scattering with neutral gas atoms along the propagation distance can be written as [66]

$$
\frac{\Delta \epsilon_{n}}{\Delta z}=8 \pi \beta_{p} n_{g 0} \frac{Z_{g}^{2} r_{e}^{2}}{\beta^{3} \gamma} \ln \left(204 Z_{g}^{-1 / 3}\right),
$$

where $\beta_{p}$ is the projected betatron focusing function in either of the transverse planes, $n_{g 0}$ is the gas density, $r_{e}=$ $2.8 \times 10^{-15} \mathrm{~m}$ is the classical electron radius, and $Z_{g}$ is the atomic number of the gas. Multiple scattering of an electron beam with $E=5.8 \mathrm{GeV}, \epsilon_{n}=300 \mathrm{~nm}$, and a xenon gas with density of $n_{\mathrm{Xe}}=10^{17} \mathrm{~cm}^{-3}\left(Z_{g}=54\right)$ would lead to an emittance growth of $[(\Delta \epsilon) / \epsilon]=7 \times 10^{-3}$.

The estimated emittance growth may safely be neglected and can be further decreased for the proposed operational gas densities of $n_{g 0}<10^{14} \mathrm{~cm}^{-3}$ and over an interaction length of a few hundred micrometer. Generation of plasma wakefields by the electron beam was analyzed as well. From linear theory [45,67], the optimal plasma density for the wake generation corresponds to $k_{\mathrm{pe}} \sigma_{z} \sim 1$, where $k_{\mathrm{pe}}=\omega_{\mathrm{pe}} / c=\sqrt{n_{\mathrm{pe}} e^{2} / m \varepsilon_{0} c^{2}}$, with $\omega_{\mathrm{pe}}$ the plasma electron frequency and $n_{\mathrm{pe}}$ the plasma electron density. A wakefield is generated for an electron-beam duration of order of the plasma skin depth; for beams with rms duration of $30 \mathrm{fs}$, wake generation is maximized for a plasma density of $4 \times 10^{17} \mathrm{~cm}^{-3}$. In order for the electron-beam self-field to dominate the ionization process, the plasma density, and therefore the gas density, should be sufficiently low so as to minimize the wakefield response of the plasma. In practice, this is not a stringent constraint, as the gas density can be optimally chosen to minimize the resulting plasma density for a given ionization yield while ensuring that sufficient ions are being produced according to the requirements of the detection system. An estimate can be obtained using the maximum field generated in the plasma at the trailing edge of the electron bunch. For simplicity, we could use the maximum value of the transverse field of the ion column at the edge of the beam. This maximum field multiplied by the duration of the bunch can give the maximum change in the transverse momentum of the beam electrons. One must operate at a density such that the increased beam divergence from the ion column is much less than the divergence due to the focused beam emittance. In practice, for ultrashort bunches that are tightly focused, operating at sufficiently low density $\left(n_{g 0}<10^{14} \mathrm{~cm}^{-3}\right)$, where the plasma wavelength is long compared to the length of the gas jet plume (typically a few $100 \mu \mathrm{m}$ ), wakefield effects can be neglected.

\section{CONCLUDING REMARKS}

In conclusion, we have shown that a lower bound on the beam charge density of intense particle beams can be determined on a single shot basis by measuring the detailed dynamics of plasmas produced using field-induced ionization of a gas through which they propagate. Using both analytical theory and detailed particle-in-cell simulations, case studies were analyzed for electron beams produced by both conventional and advanced accelerators, for various initial neutral gas densities and different gas species. In a low-density regime where a few single ions are generated, the detection method would be very similar to gas-based diagnostics for x-ray photon beams. However, when significant numbers of ions are generated (i.e., high charge density regime and/or high gas density operations), the resulting dense ion column that is generated by the electron beam can undergo Coulomb explosion due to the fact that the space-charge field of the electron beam can impart a high kinetic energy to the ionization-produced electrons that then rapidly leave the interaction region. This ion space-charge field will impact the kinetic energy 
distribution of the ions as seen by, for example, an ion timeof-flight detector. This behavior is unique to the use of electron beams as opposed to photons in this ionizationbased diagnostic. It should also be noted that the photoinduced ionization techniques used to monitor the performance of FEL radiation can provide information for retrieving particle beam distributions. The concept elaborated on in this paper enables direct probing of particle beam densities at facilities such as colliders where photonbased monitors may not be available. By measuring the ion yield, kinetic energy distribution, and spatial density distribution of the ion plasma for different neutral gas pressures and gas species, the peak electric field of the electron beams can be measured and optimized. In the regime where these intense unipolar electron beams are short compared to the quantum-mechanical tunneling timescales, ionization quenching may be observed. Contributions to the background signal on the detector from ions generated via impact ionization of the neutral gas, as well as from photoionization due to incoherent betatron emission in the case of laser plasma accelerated electron beams, were estimated. Whereas the former can contribute to a background signal for low charge density electron beams, the latter is negligible for all practical cases. For sufficiently low initial gas densities, where wakefield effects in the resultant plasma and emittance growth due to scattering are minimal, the diagnostic was found to be largely noninvasive for the primary electron beam. Because of the exponential dependency of the ionization probability on the electric field strength, it was shown that the method has the potential to measure electron beams with micron spatial and temporal timescales with unprecedented resolution at the few-nanometer and subfemtosecond scales, respectively. As such, it provides a powerful new approach to characterizing particle beams with performance parameters beyond what today's beam diagnostics can provide.

\section{ACKNOWLEDGMENTS}

One of the authors (R. T.) would like to acknowledge M. Baldinger, S. Bettoni, T. Garvey, H. P. Gehrig, R. Ischebeck, P. Juranic, Z. Ollmann, E. Prat, S. Reiche, V. Schlott, J. Smith (Tech-X), and P. Wurz for stimulating discussions and interests in this work. R. T. gives special thanks to P. Krejcik for his support to the initiative to implement this technique for the LCLS beamline and to Y. Ding and E. Prat for providing particle distributions. The work was supported by European Union's Seventh Framework Programme (FP7/2007-2011) under the Marie Skłodowska-Curie Grant Agreement No. 290605 (PSI-FELLOW/COFOUND), the Swiss National Science Foundation under Contract No. 200020:165686, and the Office of Science, Office of HEP, U.S. DOE under Contract No. DE-AC02-05CH11231.
[1] S. Doniach, Studies of the Structure of Matter with Photons from an X-ray Free-Electron Laser, J. Synchrotron Radiat. 3, 260 (1996).

[2] R. Neutze, R. Wouts, D. van der Spoel, E. Weckert, and J. Hajdu, Potential for Biomolecular Imaging with Femtosecond X-ray Pulses, Nature (London) 406, 752 (2000).

[3] H. N. Chapman, A. Barty, M. J. Bogan, S. Boutet, M. Frank, S. P. Hau-Riege, S. Marchesini, B. W. Woods, S. Bajt, W. H. Benner, R. A. London, E. Plonjes, M. Kuhlmann, R. Treusch, S. Dusterer, T. Tschentscher, J. R. Schneider, E. Spiller, T. Moller, C. Bostedt et al., Femtosecond Diffractive Imaging with a Soft-X-ray Free-Electron Laser, Nat. Phys. 2, 839 (2006).

[4] H. T. Lemke, C. Bressler, L. X. Chen, D. M. Fritz, K. J. Gaffney, A. Galler, W. Gawelda, K. Haldrup, R. W. Hartsock, H. Ihee, J. Kim, K. H. Kim, J. H. Lee, M. M. Nielsen, A. B. Stickrath, W. Zhang, D. Zhu, and M. Cammarata, Femtosecond X-ray Absorption Spectroscopy at a Hard $X$-ray Free Electron Laser: Application to Spin Crossover Dynamics, J. Phys. Chem. A 117, 735 (2013).

[5] R. Bonifacio, C. Pellegrini, and L. M. Narducci, Collective Instabilities and High-Gain Regime in a Free Electron Laser, Opt. Commun. 50, 373 (1984).

[6] M. Altarelli, R. Brinkmann, M. Chergui, W. Decking, B. Dobson, S. Düsterer, G. Grübel, W. Graeff, H. Graafsma, J. Hajdu, J. Marangos, J. Plüger, H. Reldin, D. Riley, I. Robinson, J. Rossbach, A. Schwarz, K. Tiedtke, T. Tschentscher, I. Vartaniants et al., XFEL, The European $X$-ray Free-Electron Laser, Technical Design Report DESY (2006).

[7] B. Faatz, M. Braune, O. Hensler, K. Honkavaara, R. Kammering, M. Kuhlmann, E. Ploenjes, J. RoenschSchulenburg, E. Schneidmiller, S. Schreiber, K. Tiedtke, M. Tischer, R. Treusch, M. Vogt, W. Wurth, J. Zemella, and M. Yurkov, The FLASH Facility: Advanced Options for FLASH2 and Future Perspectives, Appl. Sci. 7, 1114 (2017).

[8] P. Emma, R. Akre, J. Arthur, R. Bionta, C. Bostedt, J. Bozek, A. Brachmann, P. Bucksbaum, R. Coffee, F. J. Decker, Y. Ding, D. Dowell, S. Edstrom, A. Fisher, J. Frisch, S. Gilevich, J. Hastings, G. Hays, Ph. Hering, Z. Huang et al., First Lasing and Operation of an AngstromWavelength Free-Electron Laser, Nat. Photonics 4, 641 (2010).

[9] D. Pile, X-rays: First Light from SACLA, Nat. Photonics 5, 456 (2011).

[10] Paul Scherrer Institute, SwissFEL-Conceptual Design Report, DESY Technical Design Report (2010).

[11] C. B. Schroeder, E. Esarey, and W. P. Leemans, Beamstrahlung Considerations in Laser-Plasma-Accelerator-Based Linear Colliders, Phys. Rev. ST Accel. Beams 15, 051301 (2012).

[12] C. Behrens, F. J. Decker, Y. Ding, V. A. Dolgashev, J. Frisch, Z. Huang, P. Krejcik, H. Loos, A. Lutman, T. J. Maxwell, J. Turner, J. Wang, M. H. Wang, J. Welch, and J. $\mathrm{Wu}$, Few-Femtosecond Time-Resolved Measurements of Xray Free-Electron Lasers, Nat. Commun. 5, 3762 (2014).

[13] G. Berden, S. P. Jamison, A. M. MacLeod, W. A. Gillespie, B. Redlich, and A.F.G. van der Meer, Electro-Optic Technique with Improved Time Resolution for Real-Time, 
Nondestructive, Single-Shot Measurements of Femtosecond Electron Bunch Profiles, Phys. Rev. Lett. 93, 114802 (2004).

[14] W. P. Leemans, C. G. R. Geddes, J. Faure, Cs. Tóth, J. van Tilborg, C. B. Schroeder, E. Esarey, G. Fubiani, D. Auerbach, B. Marcelis, M. A. Carnahan, R. A. Kaindl, J. Byrd, and M. C. Martin, Observation of Terahertz Emission from a Laser-Plasma Accelerated Electron Bunch Crossing a Plasma-Vacuum Boundary, Phys. Rev. Lett. 91, 074802 (2003).

[15] J. van Tilborg, C. B. Schroeder, C. V. Filip, Cs. Tóth, C. G. R. Geddes, G. Fubiani, R. Huber, R. A. Kaindl, E. Esarey, and W. P. Leemans, Temporal Characterization of Femtosecond Laser-Plasma-Accelerated Electron Bunches Using Terahertz Radiation, Phys. Rev. Lett. 96, 014801 (2006).

[16] A. Buck, M. Nicolai, K. Schmid, C. M. S. Sears, A. Savert, J. M. Mikhailova, F. Krausz, M. C. Kaluza, and L. Veisz, Real-Time Observation of Laser-Driven Electron Acceleration, Nat. Phys. 7, 543 (2011).

[17] O. Lundh, J. Lim, C. Rechatin, L. Ammoura, A. Ben-Ismail, X. Davoine, G. Gallot, J. Goddet, E. Lefebvre, V. Malka, and J. Faure, Few Femtosecond, Few Kiloampere Electron Bunch Produced by a Laser-Plasma Accelerator, Nat. Phys. 7219 (2011).

[18] J. Fabiańska, G. Kassier, and T. Feurer, Split Ring Resonator based THz-Driven Electron Streak Camera Featuring Femtosecond Resolution, Sci. Rep. 4, 5645 (2015).

[19] R. Ischebeck, E. Prat, V. Thominet, and C. Ozkan Loch, Transverse Profile Imager for Ultrabright Electron Beams, Phys. Rev. ST Accel. Beams 18, 082802 (2015).

[20] G. L. Orlandi, P. Heimgartner, R. Ischebeck, C. Ozkan Loch, S. Trovati, P. Valitutti, V. Schlott, M. Ferianis, and G. Penco, Design and Experimental Tests of Free Electron Laser Wire Scanners, Phys. Rev. Accel. Beams 19, 092802 (2016).

[21] W. P. Leemans, R. W. Schoenlein, P. Volfbeyn, A. H. Chin, T. E. Glover, P. Balling, M. Zolotorev, K. J. Kim, S. Chattopadhyay, and C. V. Shank, X-ray Based Subpicosecond Electron Bunch Characterization Using 900 Thomson Scattering, Phys. Rev. Lett. 77, 4182 (1996).

[22] P. Tenenbaum and T. Shintake, Measurement of Small Electron-Beam Spots, Annu. Rev. Nucl. Part. Sci. 49, 125 (1999).

[23] J. Rees, Using Ions to Probe the Transverse Size of a Bunch, Report No. SLAC/AP-24, 1984.

[24] J. Buon, F. Couchot, J. Jeanjean, F. Le Diberder, V. Lepeltier, H. N. Ngoc, J. P.-y. Jorba, P. Puzo, and P. Chen, A Beam Size Monitor for the Final Focus Test Beam, Nucl. Instrum. Methods Phys. Res., Sect. A 306, 93 (1991).

[25] T. C. Katsouleas, J. Yoshii, W. B. Mori, C. Joshi, and C. Clayton, A Beam Size Monitor Based on Appearance Intensities for Multiple Gas Ionization, AIP Conf. Proc. 335, 356 (1995).

[26] A. Murokh, Tunneling Ionization Bunch Length Monitor for the Ultrarelativistic Compressed Electron Beams, Proceedings of the 2003 Particle Accelerator Conference (2003).

[27] K. Tiedtke, J. Feldhaus, U. Hahn, U. Jastrow, T. Nunez, T. Tschentscher, S. V. Bobashev, A. A. Sorokin, J. B. Hastings, S. Möller, L. Cibik, A. Gottwald, A. Hoehl, U. Kroth, M. Krumrey, H. Schöppe, G. Ulm, and M. Richter, Gas
Detectors for X-ray Lasers, J. Appl. Phys. 103, 094511 (2008).

[28] A. Adelmann, B. Hermann, R. Ischebeck, M. C. Kaluza, U. Locans, N. Sauerwein, and R. Tarkeshian, Real-Time Tomography of Gas-Jets with a Wollaston Interferometer, Appl. Sci. 8, 443 (2018).

[29] D. L. Bruhwiler, D. A. Dimitrov, J. R. Cary, E. Esarey, W. Leemans, and R. E. Giacone, Particle-in-Cell Simulations of Tunneling Ionization Effects in Plasma-Based Accelerators, Phys. Plasmas 10, 2022 (2003).

[30] C. L. O'Connell, C. D. Barnes, F.-J. Decker, M. J. Hogan, R. Iverson, P. Krejcik, R. Siemann, D. R. Walz, C. E. Clayton, C. Huang, D. K. Johnson, C. Joshi, W. Lu, K. A. Marsh, W. Mori, M. Zhou, S. Deng, T. Katsouleas, P. Muggli, and E. Oz, Plasma Production via Field Ionization, Phys. Rev. ST Accel. Beams 9, 101301 (2006).

[31] E. Oz, S. Deng, T. Katsouleas, P. Muggli, C. D. Barnes, I. Blumenfeld, F. J. Decker, P. Emma, M. J. Hogan, R. Ischebeck, R. H. Iverson, N. Kirby, P. Krejcik, C. O' Connell, R. H. Siemann, D. Walz, D. Auerbach, C. E. Clayton, C. Huang, D. K. Johnson et al., Ionization-Induced Electron Trapping in Ultrarelativistic Plasma Wakes, Phys. Rev. Lett. 98, 084801 (2007).

[32] N. Vafaei-Najafabadi, K. A. Marsh, C. E. Clayton, W. An, W. B. Mori, C. Joshi, W. Lu, E. Adli, S. Corde, C. I. Clarke, M. Litos, S. Z. Green, S. Gessner, J. Frederico, A. S. Fisher, $\mathrm{Z}$. $\mathrm{Wu}, \mathrm{D}$. Walz, and M. J. Hogan, Limitation on the Accelerating Gradient of a Wakefield Excited by an Ultrarelativistic Electron Beam in Rubidium Plasma, Phys. Rev. Accel. Beams 19, 101303 (2016).

[33] B. M. Penetrante and J. N. Bardsley, Residual Energy in Plasmas Produced by Intense Subpicosecond Lasers, Phys. Rev. A 43, 3100 (1991).

[34] F. A. Ilkov, J. E. Decker, and S. L. Chin, Reconstruction of Atomic Ionization Probabilities in Intense Laser Fields, J. Phys. B 25, 4005 (1992).

[35] D. Bauer, Ejection Energy of Photoelectrons in StrongField Ionization, Phys. Rev. A 55, 2180 (1997).

[36] P. Emma, K. Bane, M. Cornacchia, Z. Huang, H. Schlarb, G. Stupakov, and D. Walz, Femtosecond and Subfemtosecond $X$-ray Pulses from a Self-Amplified Spontaneous-Emission Based Free-Electron Laser, Phys. Rev. Lett. 92, 074801 (2004).

[37] E. Prat (private communication).

[38] A. A. Zholents and W. M. Fwaley, Proposal for Intense Attosecond Radiation from an X-ray Free-Electron Laser, Phys. Rev. Lett. 92, 224801 (2004).

[39] VSim, Tech-X, www.txcorp.com/vsim.

[40] WARP, LBNL, https://hifweb.lbl.gov/Warp/.

[41] M. V. Ammosov, N. B. Delone, and V. P. Krainov, Tunnel Ionization of Complex Atoms and of Atomic Ions in an Alternating Electromagnetic Field, JETP Lett. 64, 1191 (1986).

[42] M. Chen, E. Cormier-Michel, C. G. R. Geddes, D. L. Bruhwiler, L. L. Yu, E. Esarey, C. B. Schroeder, and W. P. Leemans, Numerical Modeling of Laser Tunneling Ionization in Explicit Particle-in-Cell Codes, J. Comput. Phys. 236, 220 (2013).

[43] W. P. Leemans, A. J. Gonsalves, H.-S. Mao, K. Nakamura, C. Benedetti, C. B. Schroeder, Cs. Tóth, J. Daniels, D. E. 
Mittelberger, S. S. Bulanov, J.-L. Vay, C. G. R. Geddes, and E. Esarey, Multi-GeV Electron Beams from Capillary-Discharge-Guided Subpetawatt Laser Pulses in the Self-Trapping Regime, Phys. Rev. Lett. 113, 245002 (2014).

[44] J. van Tilborg, S. Steinke, C. G. R. Geddes, N. H. Matlis, B. H. Shaw, A. J. Gonsalves, J. V. Huijts, K. Nakamura, J. Daniels, C. B. Schroeder, C. Benedetti, E. Esarey, S. S. Bulanov, N. A. Bobrova, P. V. Sasorov, and W. P. Leemans, Active Plasma Lensing for Relativistic Laser-PlasmaAccelerated Electron Beams, Phys. Rev. Lett. 115, 184802 (2015).

[45] E. Esarey, C. B. Schroeder, and W. P. Leemans, Physics of Laser-Driven Plasma-Based Electron Accelerators, Rev. Mod. Phys. 81, 1229 (2009).

[46] J. B. Rosenzweig, B. Breizman, T. Katsouleas, and J. J. Su, Acceleration and Focusing of Electrons in TwoDimensional Nonlinear Plasma Wake Fields, Phys. Rev. A 44, R6189 (1991).

[47] A. Pukhov and M. ter Vehn, Laser Wake Field Acceleration: The Highly Non-linear Broken-Wave Regime, Appl. Phys. B 74, 355 (2002).

[48] P. N. Juranić, M. Martins, J. Viefhaus, S. Bonfigt, L. Jahn, M. Ilchen, S. Klumpp, and K. Tiedtke, Using I-TOF Spectrometry to Measure Photon Energies at FELs, J. Instrum. 4, P09011 (2009).

[49] N. Berrah, J. Bozek, J. T. Costello, S. Düsterer, L. Fang, J. Feldhaus, H. Fukuzawa, M. Hoener, Y. H. Jiang, P. Johnsson, E. T. Kennedy, M. Meyer, R. Moshammer, P. Radcliffe, M. Richter, A. Rouzée, A. Rudenko, A. A. Sorokin, K. Tiedtke, K. Ueda, J. Ullrich, and M. J. J. Vrakking, Non-linear Processes in the Interaction of Atoms and Molecules with Intense EUV and X-ray Fields from SASE Free Electron Lasers (FELs), J. Mod. Opt. 57, 1015 (2010).

[50] B. Rudek, S.-K. Son, L. Foucar, S. W. Epp, B. Erk, R. Hartmann, M. Adolph, R. Andritschke, A. Aquila, N. Berrah, C. Bostedt, J. Bozek, N. Coppola, F. Filsinger, H. Gorke, T. Gorkhover, H. Graafsma, L. Gumprecht, A. Hartmann, G. Hauser et al., Ultra-efficient Ionization of Heavy Atoms by Intense X-ray Free-Electron Laser Pulses, Nat. Photonics 6, 858 (2012).

[51] P. B. Corkum, N. H. Burnett, and F. Brunel, AboveThreshold Ionization in the Long-Wavelength Limit, Phys. Rev. Lett. 62, 1259 (1989).

[52] W. P. Leemans, C. E. Clayton, W. B. Mori, K. A. Marsh, P. K. Kaw, A. Dyson, C. Joshi, and J. M. Wallace, Experiments and Simulations of Tunnel-Ionized Plasmas, Phys. Rev. A 46, 1091 (1992).

[53] E. Esarey, P. Sprangle, J. Krall, and A. Ting, Self-Focusing and Guiding of Short Laser Pulses in Ionizing Gases and Plasma, IEEE J. Quantum Electron. 33, 1879 (1997).

[54] U. Frühling, M. Wieland, M. Gensch, T. Gebert, B. Schütte, M. Krikunova, R. Kalms, F. Budzyn, O. Grimm, J. Rossbach, E. Plönjes, and M. Drescher, Single-Shot Terahertz-Field-Driven X-ray Streak Camera, Nat. Photonics 3, 523 (2009).

[55] P. N. Juranić, A. Stepanov, R. Ischebeck, Schlot V., C. Pradervand, L. Patthey, M. Radović, I. Gorgisyan, L. Rivkin, C. P. Hauri, B. Monoszlai, R. Ivanov, P. Peier,
J. Liu, T. Togashi, S. Owada, K. Ogawa, T. Katayama, M. Yabashi, and R. Abela, High-Precision X-ray FEL Pulse Arrival Time Measurements at SACLA by a THz Streak Camera with Xe Clusters, Opt. Express 22, 30004 (2014).

[56] I. Gorgisyan, R. Ischebeck, E. Prat, S. Reiche, L. Rivkin, and P. Juranić, Simulation of FEL Pulse Length Calculation with THz Streaking Method, J. Synchrotron Radiat. 23, 643 (2016).

[57] S. Wang, C. E. Clayton, B. E. Blue, E. S. Dodd, K. A. Marsh, W. B. Mori, C. Joshi, S. Lee, P. Muggli, T. Katsouleas, F. J. Decker, M. J. Hogan, R. H. Iverson, P. Raimondi, D. Walz, R. Siemann, and R. Assmann, X-ray Emission from Betatron Motion in a Plasma Wiggler, Phys. Rev. Lett. 88, 135004 (2002).

[58] E. Esarey, B. A. Shadwick, P. Catravas, and W. P. Leemans, Synchrotron Radiation from Electron Beams in PlasmaFocusing Channels, Phys. Rev. E 65, 056505 (2002).

[59] A. Rousse, K. T. Phuoc, R. Shah, A. Pukhov, E. Lefebvre, V. Malka, S. Kiselev, F. Burgy, J. P. Rousseau, D. Umstadter, and D. Hulin, Production of a keV X-ray Beam from Synchrotron Radiation in Relativistic Laser-Plasma Interaction, Phys. Rev. Lett. 93, 135005 (2004).

[60] K. TaPhuoc, S. Corde, R. Shah, F. Albert, R. Fitour, J. P. Rousseau, F. Burgy, B. Mercier, and A. Rousse, Imaging Electron Trajectories in a Laser-Wakefield Cavity Using Betatron X-ray Radiation, Phys. Rev. Lett. 97, 225002 (2006).

[61] G. R. Plateau, C. G. R. Geddes, D. B. Thorn, M. Chen, C. Benedetti, E. Esarey, A. J. Gonsalves, N. H. Matlis, K. Nakamura, C. B. Schroeder, S. Shiraishi, T. Sokollik, J. van Tilborg, Cs. Toth, S. Trotsenko, T. S. Kim, M. Battaglia, Th. Stöhlker, and W. P. Leemans, Low-Emittance Electron Bunches from a Laser-Plasma Accelerator Measured Using Single-Shot X-ray Spectroscopy, Phys. Rev. Lett. 109, 064802 (2012).

[62] S. Corde, K. Ta Phuoc, G. Lambert, R. Fitour, V. Malka, A. Rousse, A. Beck, and E. Lefebvre, Femtosecond X Rays from Laser-Plasma Accelerators, Rev. Mod. Phys. 85, 1 (2013).

[63] M. Litos and S. Corde, Betatron Radiation from a Beam Driven Plasma Source, AIP Proc. 1507, 705 (2012).

[64] S. T. Perkins, D. E. Cullen, and S. M. Seltzer, Tables and Graphs of Electron-Interaction Cross Sections from $10 \mathrm{eV}$ to $100 \mathrm{GeV}$ Derived from the LLNL Evaluated Electron Data Library (EEDL), Z = 1-100, LLNL Report UCRL50400 (1991).

[65] J. B. West and J. Morton, Absolute Photoionization CrossSection Tables for Xenon in the VUV and the Soft X-ray Regions, At. Data Nucl. Data Tables 22, 103 (1978).

[66] M Reiser, Theory and Design of Charged Particle Beams (Wiley, New York, 2008).

[67] S. Lee, T. Katsouleas, R. Hemker, and W. B. Mori, Simulations of a Meter-Long Plasma Wakefield Accelerator, Phys. Rev. E 61, 7014 (2000).

[68] See Supplemental Material at http://link.aps.org/ supplemental/10.1103/PhysRevX.8.021039 for details about movies. 